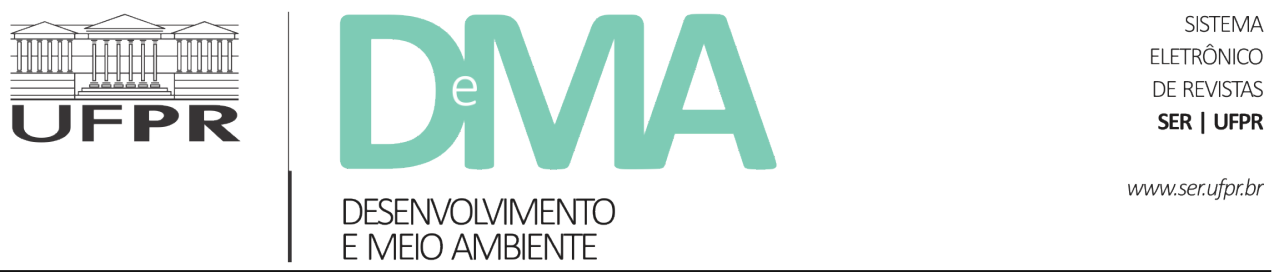

\title{
Maintaining carbon stocks in extractive reserves in Brazilian Amazonia
}

\section{Manutenção de estoques de carbono em Reservas Extrativistas na Amazônia brasileira}

\author{
Philip Martin FEARNSIDE ${ }^{1 *}$, Euler Melo NOGUEIRA² ${ }^{2}$ Aurora Miho YANAI ${ }^{1}$ \\ ${ }^{1}$ Instituto Nacional de Pesquisas da Amazônia (INPA), Manaus, AM, Brasil. \\ ${ }^{2}$ Centro Universitário UniFG, Guanambi, BA, Brasil. \\ *E-mail of contact: pmfearn@inpa.gov.br
}

Article received in April 7, 2018, final version accepted in August 30, 2018.

ABSTRACT: Extractive reserves in the Amazon Forest maintain carbon stocks out of the atmosphere, thereby avoiding greenhouse-gas emissions that provoke global warming. This and other environmental services, such as recycling water and maintaining biodiversity, provide major reasons for creating these reserves and for according them priority in government programs. The importance of reducing carbon emissions from deforestation has been the principal motivation for international funding, which has been key to creating and supporting extractive reserves, notably in the cases of Germany through the PPG7 program and Norway through the Amazon Fund. Estimating the amount of carbon in these reserves and the losses that have occurred from deforestation is essential as an input to making decisions that affect current and potential future extractive reserves. By 2014, there were 47 federal extractive reserves in Brazil's Legal Amazonia region, of which 45 were in the Amazonian Tropical Forest Biome and 26 extractive reserves belonged to states, all of which were in the Amazonia Biome. This study provides data for each of the 73 extractive reserves in Legal Amazonia, based on biomass information by forest type calculated from RadamBrasil survey data, and deforestation from PRODES monitoring by LANDSAT or equivalent satellites (30-m resolution). The stocks represent carbon in the "pre-modern" biomass, that is, the biomass present in approximately 1970, or before substantial deforestation or logging activity in the region. The carbon losses reflect only deforestation, not degradation of forest by logging and/or fire. The total area of extractive reserves in Legal Amazonia amounted to $126,709 \mathrm{~km} 2$, of which $4301 \mathrm{~km} 2(3.4 \%)$ had been cleared by 2014. Those extractive reserves had a remaining carbon stock in forest vegetation (above and belowground) of 2.1 billion tons. The carbon lost to deforestation totaled 74.9 million tons. Avoiding further carbon loss to both deforestation and degradation needs to be a high priority for the extractivists, as it is the 
value of the forest's environmental services that has the greatest potential for providing a means of support that is increasing in value and is inherently sustainable.

Keywords: environmental services; ecosystem services; biomass; deforestation; RESEX.

RESUMO: As reservas extrativistas na Amazônia mantêm carbono fora da atmosfera, evitando assim as emissões de gases de efeito estufa que causam o aquecimento global. Este e outros serviços ambientais, tais como a reciclagem de água e a manutenção da biodiversidade, fornecem importantes motivos para a criação dessas reservas e a sua prioridade nos programas governamentais. A importância de reduzir as emissões de carbono por desmatamento tem sido a principal motivação de financiamentos internacionais, as quais têm sido fundamentais para criar e apoiar as reservas extrativistas, especialmente nos casos da Alemanha, através do programa PPG7, e da Noruega, através do Fundo Amazônia. Estimativas da quantidade de carbono e das perdas pelo desmatamento são essenciais na tomada de decisões que afetam as reservas extrativas atuais e as que possam ser criadas no futuro. Até 2014 havia 47 reservas extrativistas federais na Amazônia Legal, das quais 45 estavam no Bioma Amazônia, e havia 26 reservas extrativistas a nível estadual, todas no Bioma Amazônia. Fornecemos dados para cada uma das 73 reservas extrativistas na Amazônia Legal, com base em informações de biomassa por tipo de floresta calculadas a partir dos dados do Projeto RadamBrasil e desmatamento a partir do PRODES (imagens LANDSAT ou equivalente com resolução de $30 \mathrm{~m}$ ). Os estoques representam o carbono "prémoderno", isto é, presente em aproximadamente 1970, antes que substancial desmatamento ou atividade madeireira afetasse a região. As perdas de carbono refletem apenas o desmatamento e não a degradação da floresta por exploração madeireira e fogo. As reservas extrativistas na Amazônia Legal totalizaram 126,709 $\mathrm{km} 2$, dos quais $4301 \mathrm{~km} 2(3,4 \%)$ foram desmatadas até 2014. As reservas tinham um estoque de carbono restante na vegetação florestal (acima e abaixo do solo) de 2,1 bilhões de toneladas. O carbono perdido pelo desmatamento totalizou 74,9 milhões de toneladas. Evitar novas perdas, tanto pelo desmatamento quanto pela degradação, precisa ser uma alta prioridade para os extrativistas, pois os serviços ambientais da floresta têm o maior potencial para fornecer sustento ao longo prazo, sendo um recurso cujo valor vem aumentando e que é inerentemente sustentável.

Palavras-chave: serviços ambientais; serviços ecossistêmicos; biomassa; desmatamento; RESEX.

\section{Introduction}

Amazon forests provide environmental services that are important for the world and especially for Brazil, which will suffer the most if the forests and their services are destroyed. These services include avoiding global warming, recycling water and maintaining biodiversity (e.g., Fearnside, 1997; 2008). Here we treat the matter of forest carbon stocks, which is the top-priority environmental service for the international sources of funding that have created and supported extractive reserves and that can be expected to be key to future support. We present data on deforestation and carbon stocks in each of Brazilian Amazonia's extractive reserves and discuss how land use in these reserves is increasingly shifting from sustainable extraction of rubber and other non-timber forest products to expanding deforestation for cattle pasture. Maintaining forest and carbon stocks in extractive reserves requires reversing this trend. This will require greater social control by communities in the extractive reserves. The potential value of carbon stocks as a rationale for international support of extractive reserves 
should add to the motives for extractivists to increase their control and effectively limit deforestation in the reserves.

Creating protected areas is one of the most effective measures to ensure the maintenance of environmental services in tropical forests (Adeney et al., 2009; Veríssimo et al., 2011). In the Brazilian Amazon at least 2.2 million $\mathrm{km}^{2}$ were delimited by 2014 as 718 protected areas, which includes Conservation Units, Indigenous Lands and Quilombola Territories (Nogueira et al., 2018a). The term "Conservation Units" refers to various kinds of areas for environmental protection as defined in Brazil's National System of Conservation Units, or "SNUC," and are divided into two groups: "integral protection" and "sustainable use" (Brazil, MMA, 2000). Extractive reserves are in the "sustainable use" category, which permits the continued presence of the resident population and use of renewable natural resources such as forests in defined low-impact "sustainable" ways. "Indigenous Lands" are areas recognized as traditionally inhabited by indigenous people and are administered by the $\mathrm{Na}$ tional Foundation for the Indian (FUNAI) under the Ministry of Justice, rather than by the Ministry of Environment. "Quilombolas" are occupied by the descendants of escaped African slaves who have maintained traditional communities and who have the same rights as indigenous peoples under Brazil's constitution (Brazil, INCRA, 2015).

Historically, priority areas for establishing conservation units have mainly been determined based on ecological criteria such as indicators of biodiversity (e.g., "hotspots"), endemism, rarity, or threats to species (Fearnside, 2015).Areas protected under the presumption of reconciling conservation with the presence of traditional resident populations were generally established based on the demands of social groups, such as indigenous peoples, ribeirinhos (riverside dwellers), seringueiros (rubber tappers) and other communities that are dependent on non-timber forest products or on traditional fisheries (e.g., Sustainable Use Conservation Units, Indigenous Lands and Quilombola Territories). In all of these cases however, despite the particularities and different justifications adopted for the creation of each type of protected area, global climate change adds carbon stock as one of the arguments for maintaining and creating protected areas in the Amazon (Nogueira et al., 2018a).

The various actors involved in creating extractive reserves have different motives and priorities. The extractivists themselves clearly have as a top priority securing their claim to the land against the threat of surrounding ranchers. Improving living standards and access to basic education and health services are also important priorities for extractivists. In arguing for extractive reserves, Chico Mendes always made clear that the environmental value of maintaining the forest was also important to extractivists. Within the Brazilian government, individuals in the Ministry of Environment involved with extractive reserves vary in their priorities for creating these reserves. Reasons includes the role of the reserves as a means of maintaining environmental values such as biodiversity, their role as examples of sustainability and in providing socioeconomic benefits to extractivists. These concerns are shared by other actors, such as non-governmental environmental organizations and academic scholars who study and write about extractive reserves. However, creating and maintaining Brazil's extractive reserves has always been heavily dependent on funding from international sources, and, as compared to 
other actors, the priorities of these funders are more focused on carbon and the role of Amazon forest in global climate change. The G7 Pilot Program to Conserve the Brazilian Rain Forest (PPG7), which ran from 1992 to 2008, was a critical funder in creating Brazil's current portfolio of extractive reserves, and climate was listed as a "global benefit" of the expected role of the extractive reserves component in reducing deforestation (World Bank, 1994). The World Bank's January 1992 Rain Forest Trust Resolution that established the PPG7 states that "The overall objective of the pilot program is to...reduce Brazilian rain forests' contribution to global carbon emissions..." (World Bank, 1992). This was clearly the highest priority for the country that contributed by far the most to the program: Germany. The issue of emissions was especially important in the five years prior to the December 1997 Kyoto Protocol (Fearnside, 2001). Since Norway's donations to the Amazon Fund began in 2008, it has become an important contributor to creating and maintaining Amazonian protected areas, including extractive reserves (GEF, 2018). Effectiveness in reducing emissions has been a key element in arguing in favor of international support for Brazil's Amazon Region Protected Areas Program (ARPA), including extractive reserves (Soares-Filho, 2016; Soares-Filho et al., 2010). For Norway, who donated $93 \%$ of the total received by the Amazon Fund by 2018 , the criterion on which the success is judged is reduction of Brazil's deforestation rate, which translates into reduced carbon emissions. In 2017, as stipulated in the agreement creating the Amazon Fund, the payment was reduced by half due to the deforestation rate being on the rise (Rodrigues, 2017). The criterion is limited to the deforestation rate, not other indicators such as the living standards of forest dwellers or the sustainability of the population's economic activities. With Brazil's current draconian cutbacks of government funding for the environment, the Amazon Fund is essentially the only available source for financing measures to contain deforestation (Ortiz, 2018).

The extraction of natural products for the subsistence of traditional resident populations in the Amazon is an activity as old as the existence of traditional populations (Arruda, 1999; Homma, 2003). The various traditional forms of forest product extraction (e.g., collection of plant products, fishing and hunting) have been a centuries-old subsistence practice of traditional forest-dwelling populations. Like the traditional forms of extraction, the current extractive reserves are characterized mainly by low-technology extraction (Drummond, 1996). The definition by law of areas for extractivism does not ensure that other forms of use and production by the resident populations are not used within these areas, nor does it mean that extractive activities are exclusive to these areas. In fact, extractive activities predominate in other categories of sustainable use units (e.g., National Forests, Sustainable Development Reserves, Environmental Protection Areas) or other types of protected areas (e.g., Indigenous Lands and Quilombola Territories).

Extractive reserves have protected substantial areas of forest that would be unlikely to have protection if conservation units were created through expropriation with resettlement and compensation of the residents. The extractive reserve model avoids the social injustices inherent in such a process and maintains the communities and the traditional culture of the extractivists (Fearnside, 1989). The long-term effect of this depends on both deforestation being avoided and an avoidance of degrada- 
tion of the forest, as from logging and fire. Forest degradation can lower carbon stocks in Amazonian forest and consequently lower their benefit for avoiding global warming. Degradation is known to be taking place in some extractive reserves, and the quantification of its impact on carbon stocks is a high research priority. Unfortunately, this forest maintenance has not always been as complete as expected, and processes in progress in the reserves suggest that deforestation and degradation are both likely to increase hereafter in the absence of greater social control.
In this text, we present data on carbon in extractive reserves in the Brazilian Amazon, with new analyses from recent carbon estimates for all protected areas in Amazonia (Nogueira et al., 2018a). Here we use these refined analyses to update and synthesize previous estimates of carbon stocks in Amazonian extractive reserves (Moutinho et al., 2012). In doing so we use the definition of "extractive reserves" to be those considered by the National System of Conservation Units (Brazil, MMA, 2000).

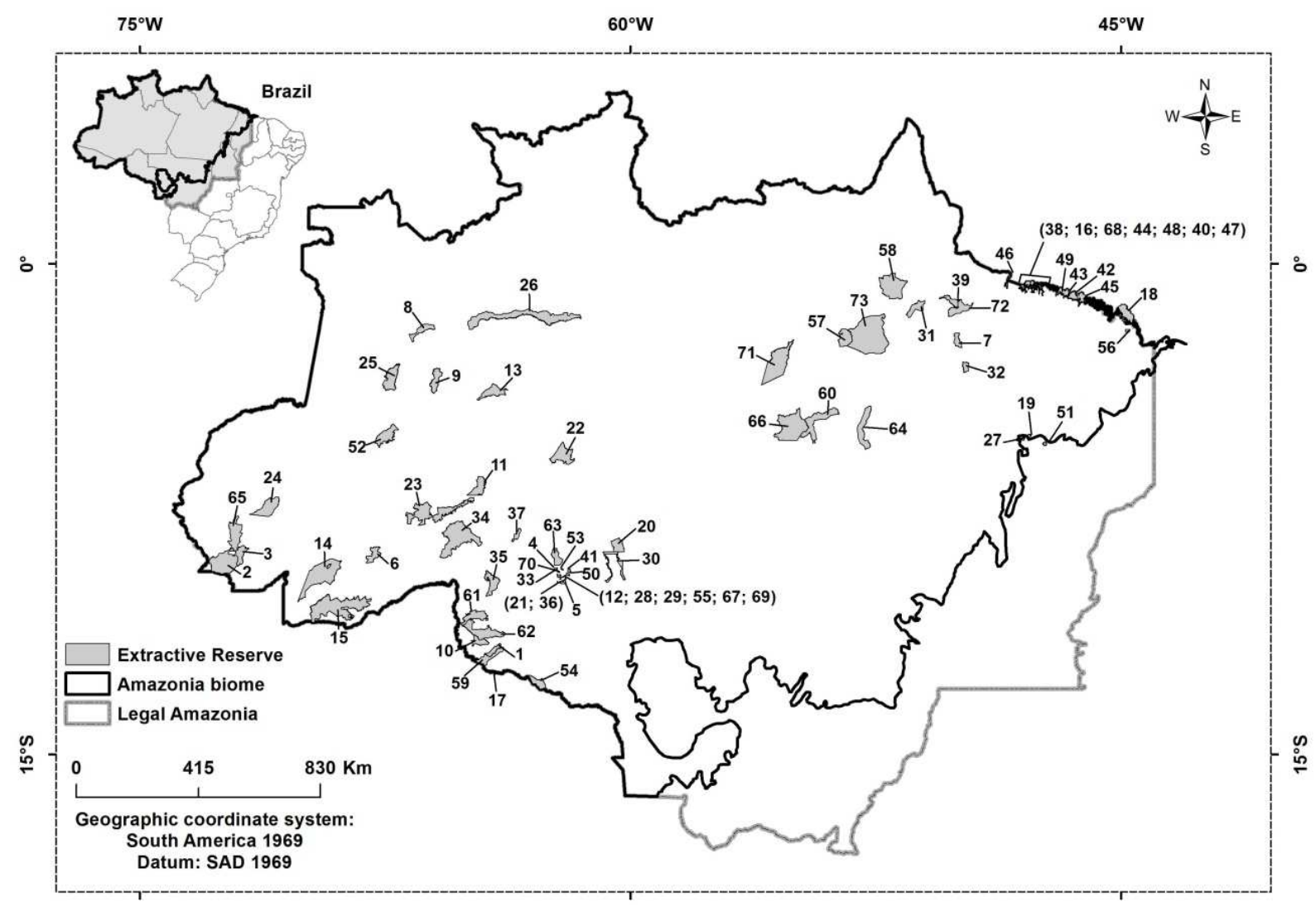

FIGURE 1 - Extractive Reserves in the Brazilian Amazonia listed up 2015 in the National Register of Conservation Units (Brazil, MMA, 2015). Reserve numbers correspond to those in Tables 1 and 2. 


\section{Methods}

The Ministry of Environment (MMA) registry (Brazil, MMA, 2015) includes 47 federal extractive reserves in Legal Amazonia (of which 45 are in the Amazonia Biome) and 26 state extractive reserves (Figure 1), all of which are in the Amazonia Biome. Legal Amazonia is a 5 million $\mathrm{km}^{2}$ administrative region, approximately $3 / 4$ of which is or was formerly covered by Amazonian forest and the remainder was covered by Cerrado savanna. The Amazonia Biome is virtually entirely contained within Legal Amazonia and includes the portion originally covered by Amazonian forest, plus enclaves of savanna within this area.

Vector maps of the extractive reserves were obtained from the Ministry of Environment database (Brazil, MMA, 2015). Spatially referenced digital maps of the each reserve were overlaid on vegetation and carbon maps, including cleared areas mapped up to 2014. Carbon estimates for each reserve were analyzed using ArcGIS ${ }^{\circledR}$ software (ESRI, 2017). See Nogueira et al. (2018a) for more details on methods.

Carbon stocks were estimated based on the biomass per hectare of each vegetation type in each reserve (Nogueira et al., 2015; 2018a). The calculation includes biomass stocks above and below ground (i.e., carbon storage in roots but not soils) of the tree and non-tree components, both alive and dead (necromass). Biomass estimates were derived mainly from interactions between volume of forest wood based on data from the RadamBrasil surveys and wood-density data (Brazil, Projeto RadamBrasil, 1973-1983; Nogueira et al., 2007). Additional biomass data, especially for forest types in southern
Amazonia, were derived by applying allometric equations (Nogueira et al., 2008a; 2008b; 2015). Original areas of each vegetation type in each reserve are estimated from the vegetation map of the Brazilian Institute of Geography and Statistics (IBGE) at a scale of 1:250,000 (Brazil, IBGE, 2012; see Nogueira et al., 2015; 2018a). Deforestation losses were determined from 2014 PRODES data (Brazil, INPE, 2016). These data are freely available on the website of the National Institute for Space Research (INPE) at 60-m resolution, which is degraded from LANDSAT-TM (30-m resolution) or equivalent satellite imagery. The lower limit for deforestation detection is 6.25 ha. Cleared areas in any enclaves of savanna vegetation in extractive reserves were computed from PMDBBS data (Brazil, IBAMA, 2015). Additional details on the carbon estimation methods can be found in Nogueira et al. (2018a).

\section{Results}

Extractive reserves in Legal Amazonia totaled $126,709 \mathrm{~km}^{2}$, of which $4301 \mathrm{~km}^{2}$ (3.4\%) had been cleared by 2014 . The area of each extractive reserve and deforestation up to 2014 are presented in Table 1 , while the original carbon stocks and the losses to deforestation are presented in Table 2. The carbon lost to deforestation added up to 74.9 million tons. Carbon estimates (both stock and loss) are for the remaining vegetation in 2014 and for the original vegetation cleared through 2014, respectively. These estimates do not consider post-clearing recovery by secondary vegetation. Table 3 summarizes the data for remaining vegetation and cleared areas and for carbon stocks and losses for federal extractive 
TABLE 1 - Cumulative clearing of vegetation by 2014 for each extractive reserve analyzed in the present study in Brazil's Legal Amazonia and Amazonia biome regions.

\begin{tabular}{|c|c|c|c|c|c|c|}
\hline $\begin{array}{l}\text { No (see } \\
\text { Fig. 1). }\end{array}$ & $\begin{array}{c}\text { Name of Protected } \\
\text { Area }\end{array}$ & $\begin{array}{c}\text { Administrative level (federal or } \\
\text { state) and protection category } \\
\text { (integral protection or sustainable } \\
\text { use)* }\end{array}$ & $\begin{array}{l}\text { Initial total } \\
\text { area** }\end{array}$ & $\begin{array}{l}\text { Area } \\
\text { covered by } \\
\text { vegetation } \\
\text { in } 2014\end{array}$ & $\begin{array}{l}\text { Cumulative } \\
\text { clearing of } \\
\text { vegetation } \\
\text { by } 2014\end{array}$ & $\begin{array}{c}\text { Area mapped } \\
\text { as deforested } \\
\text { including } \\
\text { overlapping } \\
\text { watercourses } * * * \\
\end{array}$ \\
\hline & & & \multicolumn{4}{|c|}{$\left(\right.$ in $k^{2}$ ) } \\
\hline 1 & $\begin{array}{l}\text { Reserva Extrativista } \\
\text { do Rio Cautário }\end{array}$ & FSU & 751.26 & 741.39 & 9.87 & - \\
\hline 2 & $\begin{array}{l}\text { Reserva Extrativista } \\
\text { Alto Juruá }\end{array}$ & FSU & $5,378.07$ & $5,222.58$ & 155.49 & 0.218 \\
\hline 3 & $\begin{array}{l}\text { Reserva Extrativista } \\
\text { Alto Tarauacá }\end{array}$ & FSU & $1,509.23$ & $1,473.13$ & 36.09 & 0.027 \\
\hline 4 & $\begin{array}{l}\text { Reserva Extrativista } \\
\text { Angelim }\end{array}$ & SSU & 83.84 & 76.26 & 7.59 & - \\
\hline 6 & $\begin{array}{l}\text { Reserva Extrativista } \\
\text { Arapixi }\end{array}$ & FSU & $1,337.08$ & $1,310.56$ & 26.52 & 1.965 \\
\hline 7 & $\begin{array}{l}\text { Reserva Extrativista } \\
\text { Arióca Pruanã }\end{array}$ & FSU & 838.17 & 742.89 & 95.28 & 0.153 \\
\hline 8 & $\begin{array}{l}\text { Reserva Extrativista } \\
\text { Auatí-Paraná }\end{array}$ & FSU & $1,469.49$ & $1,452.89$ & 16.60 & 0.106 \\
\hline 9 & $\begin{array}{l}\text { Reserva Extrativista } \\
\text { Baixo Juruá }\end{array}$ & FSU & $1,780.39$ & $1,754.02$ & 26.37 & 0.691 \\
\hline 10 & $\begin{array}{l}\text { Reserva Extrativista } \\
\text { Barreiro das Antas }\end{array}$ & FSU & $1,061.11$ & $1,060.51$ & 0.60 & - \\
\hline 14 & $\begin{array}{l}\text { Reserva Extrativista } \\
\text { Cazumbá-Iracema }\end{array}$ & FSU & $7,553.46$ & $7,471.24$ & 82.22 & - \\
\hline 15 & $\begin{array}{l}\text { Reserva Extrativista } \\
\text { Chico Mendes }\end{array}$ & FSU & $9,312.72$ & $8,832.33$ & 480.39 & - \\
\hline 16 & $\begin{array}{l}\text { Reserva Extrativista } \\
\text { Chocoaré-Mato } \\
\text { Grosso }\end{array}$ & FSU & 27.83 & 27.53 & 0.30 & 0.069 \\
\hline 17 & $\begin{array}{l}\text { Reserva Extrativista } \\
\text { Curralinho }\end{array}$ & SSU & 16.62 & 15.75 & 0.87 & - \\
\hline 18 & $\begin{array}{l}\text { Reserva Extrativista } \\
\text { de Cururupu }\end{array}$ & FSU & $1,572.35$ & $1,350.71$ & 221.64 & 35.255 \\
\hline
\end{tabular}




\begin{tabular}{|c|c|c|c|c|c|c|}
\hline 19 & $\begin{array}{l}\text { Reserva Extrativista } \\
\text { do Ciriáco }\end{array}$ & FSU & 81.07 & 23.82 & 57.25 & - \\
\hline 20 & $\begin{array}{l}\text { Reserva Extrativista } \\
\text { do Guariba }\end{array}$ & SSU & $1,480.84$ & $1,479.70$ & 1.14 & 0.003 \\
\hline 21 & $\begin{array}{l}\text { Reserva Extrativista } \\
\text { do Itaúba }\end{array}$ & SSU & 16.04 & 15.47 & 0.58 & - \\
\hline 22 & $\begin{array}{l}\text { Reserva Extrativista } \\
\text { do Lago do Capanã } \\
\text { Grande }\end{array}$ & FSU & $3,043.07$ & $2,998.64$ & 44.43 & 1.001 \\
\hline 23 & $\begin{array}{l}\text { Reserva Extrativista } \\
\text { do Médio Purus }\end{array}$ & FSU & $6,042.32$ & $5,998.46$ & 43.85 & 0.308 \\
\hline 24 & $\begin{array}{l}\text { Reserva Extrativista } \\
\text { do Rio Gregório }\end{array}$ & SSU & $3,069.96$ & $3,046.80$ & 23.16 & 0.026 \\
\hline 25 & $\begin{array}{l}\text { Reserva Extrativista } \\
\text { do Rio Jutaí }\end{array}$ & FSU & $2,755.13$ & $2,735.89$ & 19.24 & 0.338 \\
\hline 26 & $\begin{array}{l}\text { Reserva Extrativista } \\
\text { do Rio Unini }\end{array}$ & FSU & $8,496.85$ & $8,482.17$ & 14.68 & 2.410 \\
\hline 27 & $\begin{array}{l}\text { Reserva Extrativista } \\
\text { Extremo Norte do } \\
\text { Tocantins }\end{array}$ & FSU & 90.70 & 5.23 & 85.47 & - \\
\hline 28 & $\begin{array}{l}\text { Reserva Extrativista } \\
\text { Freijó }\end{array}$ & SSU & 6.29 & 5.39 & 0.89 & - \\
\hline 29 & $\begin{array}{l}\text { Reserva Extrativista } \\
\text { Garrote }\end{array}$ & SSU & 8.66 & 8.48 & 0.18 & - \\
\hline 30 & $\begin{array}{l}\text { Reserva Extrativista } \\
\text { Guariba-Roosevelt }\end{array}$ & SSU & $1,376.78$ & $1,303.82$ & 72.95 & 4.434 \\
\hline 31 & $\begin{array}{l}\text { Reserva Extrativista } \\
\text { Gurupá-Melgaço }\end{array}$ & FSU & $1,454.16$ & $1,430.78$ & 23.38 & 0.332 \\
\hline 32 & $\begin{array}{l}\text { Reserva Extrativista } \\
\text { Ipaú-Anilzinho }\end{array}$ & FSU & 558.34 & 375.79 & 182.55 & 0.367 \\
\hline 33 & $\begin{array}{l}\text { Reserva Extrativista } \\
\text { Ipê }\end{array}$ & SSU & 8.19 & 6.58 & 1.62 & - \\
\hline 34 & $\begin{array}{l}\text { Reserva Extrativista } \\
\text { Ituxí }\end{array}$ & FSU & $7,763.23$ & $7,746.32$ & 16.91 & 0.811 \\
\hline 35 & $\begin{array}{l}\text { Reserva Extrativista } \\
\text { Jaci-Paraná }\end{array}$ & SSU & $2,003.20$ & $1,373.32$ & 629.88 & - \\
\hline 36 & $\begin{array}{l}\text { Reserva Extrativista } \\
\text { Jatobá }\end{array}$ & SSU & 13.39 & 9.76 & 3.63 & - \\
\hline 37 & $\begin{array}{l}\text { Reserva Extrativista } \\
\text { Lago do Cuniã }\end{array}$ & FSU & 506.04 & 503.95 & 2.08 & 0.061 \\
\hline 38 & $\begin{array}{l}\text { Reserva Extrativista } \\
\text { Mãe Grande de } \\
\text { Curuçá }\end{array}$ & FSU & 335.96 & 326.71 & 9.25 & 0.462 \\
\hline 39 & $\begin{array}{l}\text { Reserva Extrativista } \\
\text { Mapuá }\end{array}$ & FSU & 937.47 & 908.35 & 29.12 & 1.061 \\
\hline
\end{tabular}




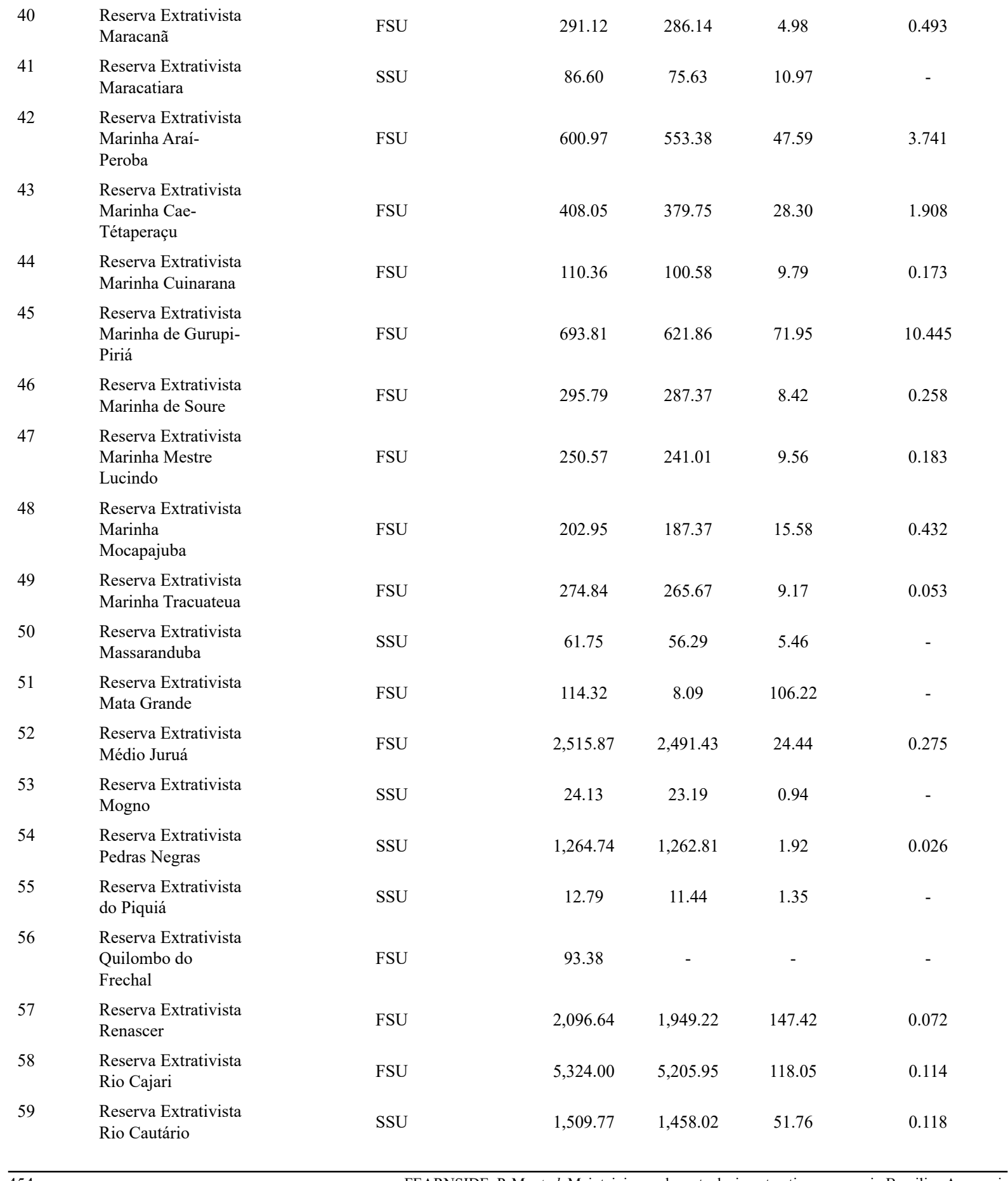




\begin{tabular}{|c|c|c|c|c|c|c|}
\hline 60 & $\begin{array}{l}\text { Reserva Extrativista } \\
\text { Rio Iriri }\end{array}$ & FSU & $3,989.88$ & $3,914.17$ & 75.71 & 1.807 \\
\hline 61 & $\begin{array}{l}\text { Reserva Extrativista } \\
\text { Rio Ouro Preto }\end{array}$ & FSU & $2,046.32$ & $1,856.73$ & 189.59 & - \\
\hline 62 & $\begin{array}{l}\text { Reserva Extrativista } \\
\text { Rio Pacaás Novos }\end{array}$ & SSU & $3,504.43$ & $3,483.40$ & 21.04 & - \\
\hline 63 & $\begin{array}{l}\text { Reserva Extrativista } \\
\text { Rio Preto-Jacundá }\end{array}$ & SSU & $1,197.67$ & $1,123.02$ & 74.66 & 0.262 \\
\hline 64 & $\begin{array}{l}\text { Reserva Extrativista } \\
\text { Rio Xingu }\end{array}$ & FSU & $3,030.01$ & $2,992.11$ & 37.90 & 3.303 \\
\hline 65 & $\begin{array}{l}\text { Reserva Extrativista } \\
\text { Riozinho da } \\
\text { Liberdade }\end{array}$ & FSU & $3,249.03$ & $3,195.23$ & 53.80 & - \\
\hline 67 & $\begin{array}{l}\text { Reserva Extrativista } \\
\text { Roxinho }\end{array}$ & SSU & 10.39 & 9.45 & 0.94 & - \\
\hline 68 & $\begin{array}{l}\text { Reserva Extrativista } \\
\text { São João da Ponta }\end{array}$ & FSU & 34.09 & 32.53 & 1.57 & 0.059 \\
\hline 69 & $\begin{array}{l}\text { Reserva Extrativista } \\
\text { Seringueira }\end{array}$ & SSU & 4.76 & 4.31 & 0.45 & - \\
\hline 70 & $\begin{array}{l}\text { Reserva Extrativista } \\
\text { Sucupira }\end{array}$ & SSU & 28.18 & 27.10 & 1.08 & - \\
\hline 71 & $\begin{array}{l}\text { Reserva Extrativista } \\
\text { Tapajós Arapiuns }\end{array}$ & FSU & $6,742.07$ & $6,226.19$ & 515.88 & 4.500 \\
\hline
\end{tabular}

* FSU = Federal Sustainable-Use conservation unit, SSU = State Sustainable Use conservation unit.

** Total area in each Extractive Reserve was calculated from vector map available from Brazil, MMA (2015). For some reserves the total area calculated from the vector maps may differ from the total area given in other official documents.

*** The estimates of carbon loss and the stock in the remaining vegetation in 2014 may, in certain reserves areas, be affected by the overlapping of classes (e.g., watercourses, forest, non-forest and deforestation), which differ between the carbon map (Nogueira et al., 2015) and the maps of the Project for Monitoring Deforestation in Amazonia (PRODES) and the Project for Monitoring Deforestation of the Brazilian Biomes by Satellite (PMDBBS) (Brazil, IBAMA, 2015; Brazil, INPE, 2016). 
TABLE 2 - Carbon estimates in extractive reserves analyzed in the present study in Brazil's Legal Amazonia and Amazonia Biome regions.

\begin{tabular}{|c|c|c|c|c|c|c|c|}
\hline \multirow[t]{2}{*}{$\begin{array}{l}\text { No. (see } \\
\text { Fig. 1). }\end{array}$} & \multirow{2}{*}{$\begin{array}{c}\text { Conservation } \\
\text { unit name } \\
\text { (from Brazil, } \\
\text { MMA, 2015) }\end{array}$} & \multirow{2}{*}{$\begin{array}{l}\text { Administrative level } \\
\text { (federal or state) and } \\
\text { protection category } \\
\text { (integral protection } \\
\text { or sustainable use)* }\end{array}$} & \multirow[t]{2}{*}{$\begin{array}{c}\text { Total area } \\
\left(\mathbf{k m}^{2}\right)^{* *}\end{array}$} & $\begin{array}{l}\text { Remaining } \\
\text { carbon stock } \\
\text { in } 2014 * * *\end{array}$ & $\begin{array}{l}\text { Carbon loss } \\
\text { by } 2014 * * *\end{array}$ & $\begin{array}{l}\text { Mean } \\
\text { remaining } \\
\text { carbon per } \\
\text { hectare in } 2014 \\
\end{array}$ & $\begin{array}{l}\text { Mean } \\
\text { carbon loss } \\
\text { per hectare }\end{array}$ \\
\hline & & & & \multicolumn{4}{|c|}{ In tons of carbon $(\mathrm{Mg} \mathrm{C})$} \\
\hline 1 & $\begin{array}{l}\text { Reserva } \\
\text { Extrativista } \\
\text { Alto Juruá }\end{array}$ & FSU & $5,378.07$ & $66,908,282.39$ & $2,141,349.71$ & 128.11 & 137.71 \\
\hline 2 & $\begin{array}{l}\text { Reserva } \\
\text { Extrativista } \\
\text { Alto Tarauacá }\end{array}$ & FSU & $1,509.23$ & $21,702,762.84$ & $605,196.66$ & 147.32 & 167.67 \\
\hline 3 & $\begin{array}{l}\text { Reserva } \\
\text { Extrativista } \\
\text { Angelim }\end{array}$ & SSU & 83.84 & $1,243,246.47$ & $124,596.56$ & 163.04 & 164.21 \\
\hline 5 & $\begin{array}{l}\text { Reserva } \\
\text { Extrativista } \\
\text { Arapixi }\end{array}$ & FSU & $1,337.08$ & $23,082,733.04$ & $427,684.81$ & 176.13 & 161.26 \\
\hline 6 & $\begin{array}{l}\text { Reserva } \\
\text { Extrativista } \\
\text { Arióca Pruanã }\end{array}$ & FSU & 838.17 & $13,327,812.99$ & $1,704,869.02$ & 179.41 & 178.93 \\
\hline 7 & $\begin{array}{l}\text { Reserva } \\
\text { Extrativista } \\
\text { Auatí-Paraná }\end{array}$ & FSU & $1,469.49$ & $26,020,305.79$ & $298,928.93$ & 179.09 & 180.10 \\
\hline 10 & $\begin{array}{l}\text { Reserva } \\
\text { Extrativista } \\
\text { Canutama }\end{array}$ & SSU & $1,979.53$ & $33,518,352.57$ & $185,156.61$ & 170.27 & 167.82 \\
\hline 11 & $\begin{array}{l}\text { Reserva } \\
\text { Extrativista } \\
\text { Castanheira }\end{array}$ & SSU & 96.61 & $1,501,989.16$ & $73,855.26$ & 163.04 & 164.46 \\
\hline 12 & $\begin{array}{l}\text { Reserva } \\
\text { Extrativista } \\
\text { Catuá-Ipixuna }\end{array}$ & SSU & $2,123.23$ & $36,518,136.66$ & $2,250,566.84$ & 183.41 & 170.23 \\
\hline 13 & $\begin{array}{l}\text { Reserva } \\
\text { Extrativista } \\
\text { Cazumbá- } \\
\text { Iracema }\end{array}$ & FSU & $7,553.46$ & $81,960,423.68$ & $1,037,123.80$ & 109.70 & 126.14 \\
\hline
\end{tabular}


Reserva

Extrativista

Chocoaré-Mato

Grosso

\section{Reserva}

16

Extrativista

Curralinho

Reserva

Extrativista de

Cururupu

18

Reserva

Extrativista do

Ciriáco

Reserva

19

Extrativista do

Guariba

20

Reserva

Extrativista do

Itaúba

Reserva

Extrativista

do Lago do

Capanã Grande

Reserva

Extrativista do

Médio Purus

Reserva

Extrativista do Rio Cautário

\section{Reserva}

Extrativista do Rio Gregório

\section{Reserva}

Extrativista do

Rio Jutaí

\section{Reserva}

Extrativista do

Rio Unini

\section{Reserva}

\section{Extrativista}

Extremo Norte

do Tocantins

Reserva

28
FSU

FSU

SSU

FSU

FSU

SSU

SSU

FSU

FSU

FSU

SSU

FSU

FSU

FSU

SSU $\begin{array}{llll}9,312.72 & 103,274,116.79 & 6,492,564.96 & 116.93\end{array}$

135.15

$\begin{array}{llll}27.83 & 300,129.52 & 3,911.72 & 109.00\end{array}$

132.24

$\begin{array}{lllll}16.62 & 253,333.48 & 12,410.76 & 160.85 & 142.02\end{array}$

$\begin{array}{lllll}1,572.35 & 5,830,764.79 & 2,410,374.22 & 43.17 & 108.75\end{array}$

$\begin{array}{lllll}81.07 & 356,954.46 & 871,643.15 & 149.88 & 152.25\end{array}$

$\begin{array}{lllll}1,480.84 & 27,360,330.39 & 20,985.73 & 184.91 & 183.97\end{array}$

$\begin{array}{lllll}16.04 & 252,093.24 & 9,477.60 & 163.00 & 163.98\end{array}$

$\begin{array}{lllll}3,043.07 & 52,820,587.02 & 702,257.75 & 702,257.75 & 176.15\end{array}$

$\begin{array}{lllll}6,042.32 & 10,529,7471.09 & 774,240.15 & 175.54 & 176.55\end{array}$

$\begin{array}{lllll}751.26 & 1,1226,638.19 & 123,392.35 & 151.43 & 125.04\end{array}$

$\begin{array}{lllll}3,069.96 & 53,994,332.52 & 402,518.08 & 177.22 & 173.78\end{array}$

$\begin{array}{lllll}2,755.13 & 48,217,575.21 & 260,006.11 & 176.24 & 135.17\end{array}$

$\begin{array}{lllll}8,496.85 & 12,3929,412.47 & 160,485.31 & 146.11 & 109.32\end{array}$

$\begin{array}{lllll}90.70 & 40,550.28 & 1,495,622.94 & 77.55 & 174.98\end{array}$

$\begin{array}{lllll}6.29 & 88,121.11 & 14,619.82 & 163.37 & 164.04\end{array}$ 
29

Reserva

Extrativista

Garrote

Reserva

30

Extrativista

Guariba-

Roosevelt

Reserva

31

Extrativista

Gurupá-

Melgaço

Reserva

32

Extrativista

Ipaú-Anilzinho

33

Reserva

Extrativista Ipê

Reserva

34

Ituxí

35

Reserva

Extrativista

Jaci-Paraná

Reserva

36

Extrativista

Jatobá

37

Reserva

Extrativista

Lago do Cuniã

Reserva

38

Extrativista

Mãe Grande de

Curuçá

Reserva

39

Mapuá

Reserva

$40 \quad$ Extrativista

Maracanã

Reserva

41

Extrativista

Maracatiara

Reserva

42

Extrativista
Extrativista

Extrativista
SSU

SSU

FSU

FSU

SSU

FSU

SSU

SSU

FSU

FSU

FSU

FSU

SSU

FSU
8.66

140951.33

2954.67

166.21

165.16

$\begin{array}{llll}1,376.78 & 20,414,313.68 & 1,137,847.42 & 156.57\end{array}$

155.97

$\begin{array}{llll}1,454.16 & 25,824,283.63 & 417,166.42 & 180.49\end{array}$

178.40

$558.34 \quad 6,720,226.33 \quad 3,296,893.84$

178.83

180.60

$\begin{array}{llll}8.19 & 107,224.94 & 264,55.61 & 163.00\end{array}$

163.65

$7,763.23 \quad 135,952,750.18$

$284,113.13$

175.51

168.02

$2,003.20 \quad 22,451,152.12 \quad 10,269,651.72 \quad 163.48$

163.04

13.39

$160,663.53$

$60,273.70$

164.56

166.26

$506.04 \quad 6,986,437.54$

$27,952.55$

138.63

134.25

Marinha Araí-

Peroba

$$
335.9
$$

$2,255,473.75$

$111,633.38$

69.04

120.72

$937.47 \quad 15,809,893.73$

496,800.61

174.05

170.62

$291.12 \quad 2,127,369.63 \quad 68,453.46$

137.43

$1,286,028.43$

$182,697.00$

170.05

166.49

$600.97 \quad 4,002,430.87$

$637,049.29$

72.33

133.87 
Reserva

43 Extrativista

Marinha Cae-

FSU

408.05

2846214.82

336005.31

74.95

118.71

Tétaperaçu

Reserva

44

Extrativista

Marinha

FSU

110.36

$1,172,922.68$

$159,676.46$

116.62

163.17

Cuinarana

Reserva

45

Extrativista

Marinha de

FSU

693.81

$4,239,732.86 \quad 783,714.68$

68.18

108.93

Gurupi-Piriá

Reserva

46

Extrativista

Marinha de

FSU

295.79

$1,721,784.17$

$110,254.96$

59.91

131.00

Soure

Reserva

47

Extrativista

Marinha Mestre

FSU

$250.57 \quad 1,859,552.18$

$121,455.12$

77.16

127.09

Lucindo

Reserva

48

Extrativista

Marinha

FSU

202.95

$1,685,593.15$

$240,203.98$

89.96

154.20

Mocapajuba

Reserva

49

Extrativista

Marinha

FSU

274.84

$2,232,085.10$

$127,398.44$

84.02

138.90

Tracuateua

Reserva

50

Extrativista

Massaranduba

SSU

$917,409.72$

$88,927.47$

162.98

162.99

Reserva

51

Extrativista

Mata Grande

FSU

114.32

121225.44

1591330.06

149.81

149.81

Reserva

52

Extrativista

Médio Juruá

FSU

$44,135,068.05$

$424,826.37$

177.15

173.83

Reserva

53

Extrativista

SSU

24.13

$378,342.46$

$15,288.81$

163.12

163.28

Mogno

SSU

$1,264.74 \quad 1,2718,751.49$

$31,791.33$

100.72

165.19

Pedras Negras

Reserva

55

Extrativista do

SSU

12.79

$211,272.68$

$24,743.53$

184.76

183.22 
Reserva

Extrativista

Quilombo do

Frechal $^{(2)}$

Reserva

Extrativista

Renascer

Reserva

Extrativista Rio

Cajari

Reserva

59

Extrativista Rio

Cautário

Reserva

60

Extrativista Rio

Iriri

Reserva

61

Extrativista Rio

Ouro Preto

Reserva

62

Extrativista Rio

Pacaás Novos

Reserva

63

Extrativista Rio

Preto-Jacundá

Reserva

64

Extrativista Rio

Xingu

Reserva

65

Extrativista

Riozinho da

Liberdade

Reserva

66

Extrativista

Riozinho do

Anfrísio

Reserva

67

Extrativista

Roxinho

Reserva

68

Extrativista São

João da Ponta

Reserva

69

Extrativista

Seringueira

Reserva

70
FSU

FSU

FSU

SSU

FSU

FSU

SSU

SSU

FSU

FSU

FSU

SSU

FSU

SSU

SSU
$93.38 \quad 1,419,198.73$

$2,096.64 \quad 31,854,327.45$

$23,80740.11$

163.42

161.50

$5,324.00 \quad 75,332,338.93$

$2,136,443.45$

144.70

180.98

$1,509.77 \quad 21,841,639.46$

$814,979.93$

149.80

157.47

$3,989.88 \quad 61,143,694.56 \quad 1,226,100.70$

156.21

161.94

$2,046.32 \quad 29,755,917.86 \quad 3,161,176.32$

160.26

166.74

$\begin{array}{llll}3,504.43 & 59,191,089.96 & 367,549.19 & 169.92\end{array}$

174.72

$\begin{array}{llll}1,197.67 & 1,8595,789.83 & 1,264,426.95 & 165.59\end{array}$

169.36

$\begin{array}{llll}3,030.01 & 45,019,486.22 & 595,846.46 & 150.46\end{array}$

157.22

$3,249.03 \quad 46,813,193.54$

$924,148.32$

146.51

171.77

$7,360.83 \quad 122,380,199.93$

$613,672.46$

167.07

171.14

10.39

$154,125.76$

$15,322.54$

163.07

163.28

34.09

$426,031.84$

$25,696.86$

130.97

164.19

$\begin{array}{llll}4.76 & 70,297.69 & 7,364.03 & 163.19\end{array}$

164.52

28.18

$441,690.93$

$17,863.84$

162.99

164.84

Sucupira 


\begin{tabular}{|c|c|c|c|c|c|c|c|}
\hline 71 & $\begin{array}{l}\text { Reserva } \\
\text { Extrativista } \\
\text { Tapajós } \\
\text { Arapiuns }\end{array}$ & FSU & $6,742.07$ & $115,546,785.37$ & $8,859,338.94$ & 185.58 & 171.73 \\
\hline 72 & $\begin{array}{l}\text { Reserva } \\
\text { Extrativista } \\
\text { Terra Grande } \\
\text { Pracuúba }\end{array}$ & FSU & $1,948.64$ & $34,156,598.13$ & $1,044,412.24$ & 180.57 & 183.19 \\
\hline 73 & $\begin{array}{l}\text { Reserva } \\
\text { Extrativista } \\
\text { Verde Para } \\
\text { Sempre }\end{array}$ & FSU & $12,893.12$ & $178,775,725.20$ & $6,993,238.69$ & 143.00 & 178.85 \\
\hline
\end{tabular}

* IL = Indigenous land, MT = Maroon territory, FSP = Federal Strictly Protected conservation unit, FSU = Federal Sustainable-Use conservation unit, SSP = State Strictly Protected conservation unit, SSU = State Sustainable Use conservation unit, MSP = Municipal Strictly Protected conservation unit, MSU = Municipal Sustainable Use conservation unit.

** Total area in each conservation unit was calculated from vector maps from Brazil, MMA (2015). Total areas calculated from the vector maps for some conservation units may differ from the areas given in official documents.

*** The estimates of carbon loss and the stock in the remaining vegetation in 2014 may, in certain reserves areas, can be affected by the overlapping of classes (e.g., hydrography, forest, non-forest and deforestation), which differ between the carbon map (Nogueira et al., 2015) and the maps of the Project for Monitoring Deforestation in Amazonia (PRODES) and the Project for Monitoring Deforestation of the Brazilian Biomes by Satellite (PMDBBS) (Brazil, IBAMA, 2015; Brazil, INPE, 2016).

(1) In these reserves it was not possible to calculate the amount of carbon stored.

(2) Carbon values refer to original carbon stocks without any carbon loss due to clearing. In these reserves a total loss of original vegetation cover may have occurred.

TABLE 3 - Remaining areas covered by original vegetation and deforested areas, together with their respective carbon stocks and losses, in extractive reserves in Brazil's Legal Amazonia region.

\begin{tabular}{|c|c|c|c|c|c|c|c|c|c|}
\hline \multirow[t]{2}{*}{$\begin{array}{c}\text { Administrative } \\
\text { level }\end{array}$} & \multirow[t]{2}{*}{$\begin{array}{c}\text { No of } \\
\text { reserves }\end{array}$} & $\begin{array}{c}\text { Initial } \\
\text { total } \\
\text { area* }\end{array}$ & $\begin{array}{c}\text { Area } \\
\text { covered } \\
\text { by vege- } \\
\text { tation in } \\
2014 \\
\end{array}$ & $\begin{array}{c}\text { Cumulative } \\
\text { clearing of vege- } \\
\text { tation by } 2014\end{array}$ & $\begin{array}{c}\text { Area mapped } \\
\text { as deforested } \\
\text { including } \\
\text { overlapping } \\
\text { watercourses* } \\
\end{array}$ & $\begin{array}{c}\text { Remaining } \\
\text { carbon stock } \\
\text { in } 2014\end{array}$ & $\begin{array}{l}\text { Carbon loss } \\
\text { by } 2014\end{array}$ & $\begin{array}{c}\text { Mean } \\
\text { remaining } \\
\text { carbon per } \\
\text { hectare in } \\
\text { forest in } 2014 \\
\end{array}$ & $\begin{array}{c}\text { Mean } \\
\text { carbon loss } \\
\text { per hectare } \\
\text { deforested }\end{array}$ \\
\hline & & \multicolumn{4}{|c|}{ In square kilometers $\left(\mathrm{km}^{2}\right)$} & \multicolumn{4}{|c|}{ In tons of carbon $(\mathrm{Mg} \mathrm{C})$} \\
\hline Federal & 47 & 106,528 & 103,210 & 3,224 & 89 & $1,794,744,514$ & $457,956,751$ & 174 & 179.8 \\
\hline $\begin{array}{c}\text { Federal }+ \\
\text { State }\end{array}$ & 73 & 126,709 & 122,314 & 4,301 & 105 & $2,053,317,615$ & $74,851,961$ & 168 & 174.0 \\
\hline
\end{tabular}

\footnotetext{
* See notes in Table 2 .
} 
reserves, state extractive reserves, and for both types together.

The original carbon density in tons per hectare $\left(\mathrm{Mg} \mathrm{ha}^{-1}\right)$ estimated before clearing occurred in the extractive reserve presented in Figure 2. The reserves had a remaining carbon stock in forest vegetation of 2.1 billion tons, with average carbon density per hectare estimated at 168 tons (Table 3 ). The average carbon density is higher in the federal than in the state extractive reserves.

\section{Discussion}

\subsection{Carbon as a foundation for maintaining forest}

Using the value of carbon stocks to maintain forests has multiple environmental "co-benefits," such as water cycling and biodiversity, as well as social benefits in regards to maintaining traditional communities and cultures (e.g., Stickler et al., 2009). The benefits to local communities, in addition to their own value, have additional importance from their role in providing motivation for development of governance that can be more effective, cheaper and socially much more attractive than the predominant means of controlling deforestation in the region through reliance on inspections and fines from government agencies. However, it is essential that this local governance actually work, as reflected in halting deforestation in the extractive reserves.

The stocks of carbon documented here represent only the first step in the long process of tapping the climatic value of the forest and transforming this value into a system that both maintains the forest and provides support to the resident population.
We certainly do not have the answers to the many challenges involved in designing and institutionalizing such a system. Some lessons can be gained from existing projects in extractive reserves to pay for environmental services or to implement projects for Reducing Emissions from Deforestation and Degradation (REDD+).

The state of Amazonas has a "Bolsa Floresta" ("Forest Stipend" or "Forest Allowance") program financed by the Amazon Fund to provide small monthly payments to families in protected areas, including extractive reserves, plus more substantial contributions to community associations and for infrastructure, including schools, solar panels and water tanks (e.g., Viana et al., 2012; Bakkegaard $\&$ Wunder, 2014). The program does not have an explicit tie to carbon, but participating families sign an agreement to limit their future clearing to the small annual amounts they have been clearing in the past. However, a test of what happens when these agreements are violated has yet to occur. The beneficiaries of the program clearly have increased wellbeing in comparison with those who live outside of protected areas, but the greatest potential benefit of the program has not yet materialized: namely stimulating traditional residents outside of protected areas to demand that government authorities create new sustainable-use protected areas so that these people can also benefit. The most critical location where this is needed is the vast area of public lands to the west of the Purus River that is now at risk from road-building plans associated with the BR-319 (Manaus-Porto Velho) Highway (Fearnside \& Graça, 2006).

Another approach is REDD+, where avoided carbon emissions would be accounted for and compensated, presumably from the voluntary 


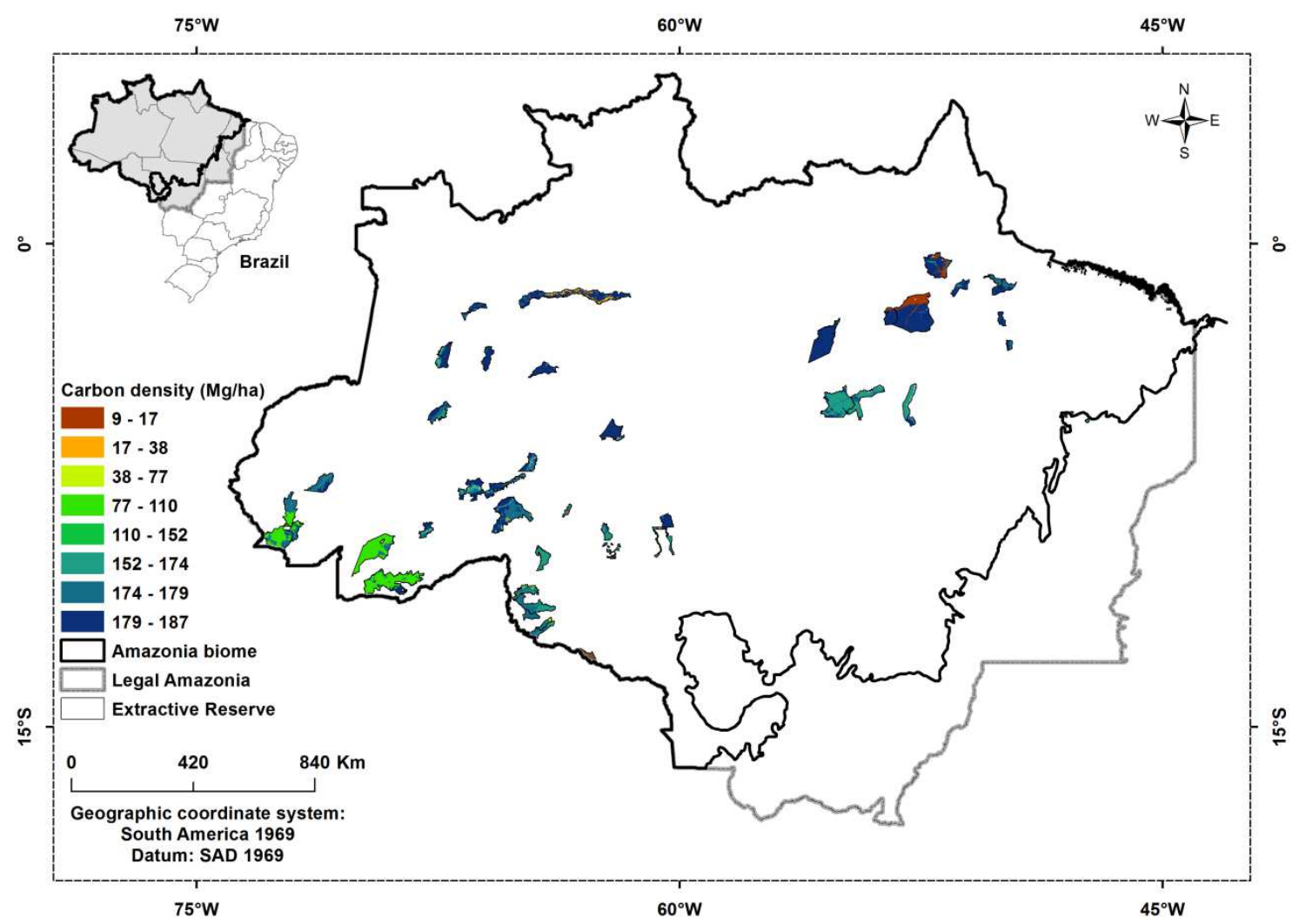

FIGURE 2 - Carbon density in tons per hectare $\left(\mathrm{Mg} \mathrm{ha}^{-1}\right)$ in the extractive reserves in the Brazilian Amazonia, estimated before clearing had occurred.

market (although in the future a REDD + mechanism is expected under the UNFCCC). REDD is an extremely controversial topic, both in Brazil and globally (Fearnside, 2012a). Carbon accounting issues that need to be addressed in order to assure that climate benefits are real include dealing with uncertainty in the measurement of carbon stocks and their changes (Fearnside, 2000), the "baseline" (reference scenario) used for attributing emissions reductions to a mitigation project (i.e., "additionality") (Yanai et al., 2012; Vitel et al., 2013), "leakage" (displacement of deforestation to locations beyond a project's boundaries) (Fearnside, 2009) and "permanence" (the time that carbon remains out of the atmosphere) (Fearnside et al., 2000; Fearnside, 2002). These issues are substantial, but all have solutions (Fearnside, 2012b; Fearnside et al., 2014). However, most opposition to REDD is not rooted in theoretical issues regarding carbon accounting, but rather in political issues regarding the distribution of financial and employment benefits (Fearnside, 2012a; 2013).

So far, extractive reserves have had a relatively minor presence among Brazil's REDD+ projects 
(e.g., Gomes, 2016). Two extractivist groups signed an anti-REDD statement in 2011: Sindicato dos Trabalhadores/as Rurais de Xapuri in Acre and Resex Renascer Tapajós-Arapiuns in Pará (Grupo Carta de Belém, 2011). One REDD+ project that is underway is the Resex Rio Preto Jacundá REDD+ Project (Biofílica Investimentos Ambientais AS, 2016). This REDD+ project follows the standard model for certified private-sector projects in the voluntary carbon market, with calculations of avoided emissions that are specific to the extractive reserve, in addition to claiming environmental and social co-benefits. In Amapá the Cajari Carbon Project is a state government initiative that also includes areas outside of the Cajari extractive reserve (IEF, 2018). The original proposal had carbon added to it to make it eligible for funding under a Petrobrás program, but essentially all of the benefits ascribed to the project by its managers are in the area of social and sustainability, without measurable links to carbon emissions (Superti \& Aubertin, 2015). In Acre the state government's Incentive System for Environmental Services (SISA) seeks to reduce the state's loss of environmental services, including carbon stocks, hydrological services and biodiversity. Like the project in Amapá, it provides infrastructure and government services that encourage non-destructive economic activities but does not make payments to stakeholders (Neves et al., 2013). The Acre program includes extractive reserves among the many land categories in the state. The program has so far been funded by the Acre state government, but a memorandum of understanding with the US state of California foresees future funding of the program from financial flows that are based on avoided carbon emissions (Palmer et al., 2017). Forest degradation in Acre is substantially increasing the state's carbon emission as compared to what was planned under SISA. A major recent degradation event was caused by forest fires during the 2015 drought that affected an area of forest larger than all of the deforestation in the state between 2004 and 2015 (da Silva et al., 2018).

\subsection{Deforestation and degradation}

The 6.25 ha lower limit for deforestation detection by PRODES may bias our results for deforestation in the extractive reserves downward more than the bias from this detection limit in deforestation estimates for other locations in Amazonia, such as settlement projects and areas dominated by large ranches. This is because the traditional clearings made in extractivist family collection areas ("colocações") are often smaller than this minimum area. In addition, clearings in colocações are scattered throughout the forest, unlike clearings in settlement projects, which are often contiguous with clearings by neighbors. Kalamandan et al. (2018) have recently shown the importance of small clearings, which are increasingly common throughout Brazilian Amazonia.

The biomass data used in this study are derived from RadamBrasil forest survey data transformed to biomass based on allometric equations, wood density and other information derived by Nogueira et al. (2008a; 2015; 2018a). Various other estimates of Amazon forest biomass exist, but they rely on much more limited ground-truth data than the almost 3000 1-ha RadamBrasil plots (see review in Fearnside, 2018). The same dataset used here for forest biomass is being used in Brazil's $3^{\text {rd }}$ National Communication to the United Nations Framework 
Convention on Climate Change (see Bustamante et al., 2018).

Amazon forest biomass varies considerably across the region as a result of a complex interaction among factors such as the physical and chemical properties of the soil, climate and the site's disturbance history, including cutting and/or enrichment done by pre-columbian human populations (Heckenberger et al., 2003; 2007; Malhi et al., 2004; 2015; Quesada et al., 2011; 2012). Forest biomass is generally the highest in central Amazonia, for example near Manaus, and the lowest in areas close to the cerrado (central Brazilian savanna) (Nogueira et al., 2015). Soils have a general gradient from high fertility areas near the Andes in the west to lower fertility in the east, while rainfall has a gradient from high precipitation and absence of a dry season in the northwestern area near Colombia, to low precipitation and long dry seasons in the southeastern portion of the region (Malhi et al., 2004; 2006). More fertile soil is associated with tree species with less-dense wood and causes trees to grow faster, thereby producing less-dense wood even within the same species (Nogueira et al., 2007). Another factor reducing biomass on more-fertile soils is trees being shorter for any given diameter (Nogueira et al., 2008b). Forests in western Amazonia have lower stature, reducing biomass (Feldpausch et al., 2012). Trees grow faster near the Andes with high soil fertility, but they also have higher mortality, which results in faster turnover but not in higher biomass (Phillips et al., 2004). Forests in portions of Amazonia that have long dry seasons have lower biomass not because of lower productivity, but rather because trees in these areas have higher mortality and shorter lifespans (Malhi et al., 2015). Non-forest vegetation can result either from clima- te, for example areas with an excessively long dry season (Hutyra et al., 2005; Salazar et al., 2007), or from very unfavorable soils such as white sand or hardpan (e.g., Lisboa, 1975). The pre-modern biomass in extractive reserves reflects the biomass in the places where they are located, but they are not evenly distributed across the region (Figure 1). Average pre-modern biomass carbon stock in extractive reserves of all types was $168 \mathrm{Mg} \mathrm{C} \mathrm{ha}^{-1}$ in 2014 (Table 3), while the average in Brazil's Legal Amazon region was $148.8 \pm 32.5 \mathrm{Mg} \mathrm{C} \mathrm{ha}^{-1}$ and $164.0 \pm 36.0 \mathrm{t} \mathrm{Mg} \mathrm{C} \mathrm{ha}^{-1}$ in the country's Amazonia Biome (Nogueira et al., 2015).

Moutinho et al. (2012, p. 134) have calculated a biomass carbon stock (above + below ground) for all Brazil's extractive reserves that had been established by 2008; values for each reserve are not presented, but total values are given by jurisdiction category, which are equivalent to average stocks of 144.2 tons per hectare $(\mathrm{Mg} \mathrm{C} \mathrm{ha-1})$ in federal reserves, $142.2 \mathrm{Mg} \mathrm{C}^{-1}$ in state reserves and 143.7 $\mathrm{Mg} \mathrm{C} \mathrm{ha}^{-1}$ considering both types together. Our estimates are 174 tons per hectare in federal reserves (20.7\% higher), $135 \mathrm{Mg} \mathrm{C} \mathrm{ha}^{-1}$ in state reserves (5.0\% lower) and $168 \mathrm{Mg} \mathrm{C} \mathrm{ha}^{-1}$ in both types together (16.9\% higher) (Table 3$)$. Moutinho et al. (2012, p. 82) used above-ground forest carbon stocks based on the map by Saatchi et al. (2007) and added $20 \%$ to these values to represent below-ground carbon based on (Houghton et al., 2000; 2001). The Saatchi et al. (2007) map was based on ground-truth information on primary forests in Brazil at only 53 distinct locations, and almost half of these had a sample areas of either $<1$ ha or of unknown area (See Fearnside, 2018). The forest biomass values used in the present study are based on the RadamBrasil measurements of trees in 2317 
plots, each 1 ha in area, while biomass in non-forest or contact zones was based on another 1830 plots and subplots of variable size (Nogueira et al., 2015).

The biomass and carbon values in Tables 1 and 2 are for "pre-modern" forests, that is, forests at the time of the RadamBrasil surveys (which were conducted mainly in the 1960s and early 1970s). The RadamBrasil surveys were done in a period when very little damage had been done to the forest by logging. Forest fires had also been much less frequent than in recent decades. Logging preferentially removes large trees, thus lowering forest biomass (Sist \& Ferreira, 2007; Mazzei et al., 2010). Even when it is done with "reduced impact," logging operations can also kill many trees that are not harvested (Sist et al., 2014). The forest today would have lower biomass because the disturbance from logging substantially increases the vulnerability of Amazon forests to fire (Uhl \& Buschbacher, 1985; Cochrane et al., 1999; Nepstad et al., 1999). This can kill more trees (Barlow et al., 2003; Vasconcelos et al., 2013) and initiate a self-reinforcing process of positive feedback characterized by successive fire and mortality events (Nepstad et al., 2001; Barlow \& Peres, 2006; Berenguer et al., 2014). In Acre and neighboring areas, fires also stimulate invasion by bamboo, further reducing forest biomass and carbon stocks (Silva et al., 2017; Nogueira et al., 2008b).

Degradation losses in extractive reserves lack quantification, as is also the case for most areas in Amazonia. Understory fires affected at least 2500 $\mathrm{km}^{2}$ in the state of Acre during the 2005 drought (Brown et al., 2006). In the Chico Mendes Extractive reserve, fire caused the number of dead stems to be much greater in burned plots than in unburned plots studied by Barlow and coworkers (2012), but the lack of plots from before the fire prevented statistically significant quantification of biomass losses from being recorded. Amazonian forest has very high natural variation in biomass between plots over short distances (Nascimento \& Laurance, 2002; Fearnside, 2018). The droughts of the type that occurred in 2005 (Marengo et al., 2008; Zeng et al., 2008; Phillips et al., 2009b) and again in 2010 (Lewis et al., 2011; Marengo et al., 2011) are expected to increase dramatically in the coming decades under projected global warming (Cox et al., 2008).

There is a tendency to view emissions from logging as "directly human-induced" (as termed in the Kyoto Protocol), but forest fire is viewed as a "natural" source. However, almost no fires are "natural." Not only are virtually all Amazonian fires caused by a human ignition source, a large proportion of them have their origin in forests that have been made susceptible to fire by logging. Fire represents a significant threat to projects that intend to reward climate benefits through Reducing Emissions from Deforestation and Degradation (REDD+) (Aragão \& Shimabukuro, 2010; Silva et al., 2013).

The carbon loss values presented in Tables 2 and 3 represent gross values and do not include reabsorption of some carbon by the deforested landscape, including secondary forest. Calculations exist for this uptake of Brazilian Amazonia as a whole, not specifically for extractive reserves. Secondary forests grow much slower if they are from degraded cattle pasture than if they are agricultural fallows (Fearnside \& Guimarães, 1996; Wandelli \& Fearnside, 2015). To the extent that extractivists are expanding cattle pastures, as in the case of the Chico Mendes Extractive Reserve in Acre, this slower rate of carbon uptake 
by secondary forest will predominate, as it does in most of Brazilian Amazonia.

In addition to its role in storing carbon, Amazon forest has also been acting as a carbon sink. In the 1990s this sink was believed to be very large, but correction of technical problems with early $\mathrm{CO}_{2}$ flux measurements made from towers has resulted in much lower estimates for the magnitude of the sink (e.g., Araújo et al., 2002). Monitoring of tree diameters in permanent plots has shown a basin-wide average uptake, although the magnitude varies among sites with the greatest increases near the Andes (Phillips et al., 2009a; Lewis et al., 2004). Estimates vary depending on methods (Grace, 2016). The sink is reversed under drought conditions (Phillips et al., 2009b; Gatti et al., 2014), and severe droughts are expected to have a marked increase with climate change (Cox et al., 2008; Latif et al., 2015). There has been a decreasing trend in recent years: based on monitoring of 321 plots (mean plot area $=1.2$ ha), the average magnitude of the Amazon forest sink has decreased from approximately $1.5 \mathrm{MgC}$ ha $^{-1}$ year $^{-1}$ in 1985 to $0.25 \mathrm{MgC} \mathrm{ha}^{-1}$ year $^{-1}$ in 2011 (Brienen et al., 2015).

\subsection{Challenges to controlling carbon loss}

Protected areas represent a bulwark against climate change, and the need to avoid greenhouse-gas emissions is likely to be an increasingly important factor in decisions on creating and supporting these areas, including extractive reserves (Nogueira et $a l ., 2018 \mathrm{~b}$ ). The effectiveness of protected areas in maintaining their carbon stocks will be critical to determining the allocation of resources in global and national efforts to fight climate change.
Logging is a delicate issue in extractive reserves because, unlike extraction of non-forest timber products like rubber and Brazilnuts, logging is not inherently sustainable unless strict limits on harvest intensity are guaranteed to be respected over the course of many human generations. This requires social controls strong enough to not be relaxed or abandoned when the forest's timber stocks are drawn down to a pre-established limit, and when both population increase and the continued rise in the individual residents' desire for material consumption translate into pressure to change or evade the forest management regulations (Fearnside, 2003).

An example of the problem in maintaining the previous patterns of non-destructive behavior that have characterized extractivists for over a century is shown by expanding areas of cattle pasture in the Chico Mendes extractive reserve in Acre (Salisbury \& Schmink, 2007; Vadjunec et al., 2009). Deforestation has increased, and a contingent of residents in the reserve has, in fact, become ranchers rather than extractivists (e.g., Salomon, 2008; Carranca, 2014). By 2014 a total of $480.4 \mathrm{~km}^{2}$ of deforestation had occurred in this reserve $(5.2 \%$ of the original forest area) (Table 1), much of it in the last few years.

The reason that extractive reserves are created and receive priority in government services as compared to unprotected areas in the interior of Brazilian Amazonia is because of the environmental services. The reason is not the fact that people in extractive reserves have a right to services such as education and health care: although extractive populations have a right to these services, so do populations outside of protected areas, and the sad fact is that, in practice, having these rights does not mean that the government will provide them in a timely fashion. The same amount of money spent 
could provide, for example, schools and health centers for many more people in one of the country's "favelas" (shantytowns) than in remote areas in the Amazon forest. The residents of extractive reserves are providing a service by maintaining the forest, and it is important that they realize this is the reason for the benefits they receive.

Rubber extraction itself is no longer lucrative enough to make extractive reserves economically viable without some form of subsidy (Jaramillo-Giraldo et al., 2017). This means that extractivists must continually demonstrate that they have social controls sufficient to avoid loss of environmental services in order to justify funds from sources that are motivated to invest in maintaining environmental services. It is a basic precept of any program for payment for environmental services that the recipients must have control over the land that provides the services (Wunder et al., 2009). Normally this control refers to land ownership, as through a land title, but in the case of an extractive reserve it would apply as an adequate level of control by the extractivist community organization over the activities that take place in the reserve (e.g., Global Compass, 2014). If individual families are free to become cattle ranchers and expand their clearings at will, the basis for transforming the climatic value of the forest's carbon stocks into a means of support for the extractivist population is undermined. While a variety of opinions exists on compensating the climate benefits of maintaining tropical forests, this compensation is likely to become an increasingly high priority if the countries of the world are serious about containing global warming (Fearnside, 2012a). Maintaining the carbon stocks documented in this study is the most visible of the environmental services upon which the future of these extractivist populations depends.

\section{Conclusions}

Extractive reserves in Brazil's Amazon region contain substantial amounts of carbon. These reserves are not immune to deforestation and to forest degradation, and maintaining their carbon stocks and associated climate benefits requires active defense. This indicates the need for a level of social control within the extractivist communities that is sufficient to prevent deforestation and forest degradation in the reserves.

\section{Acknowledgments}

The authors' research is supported by Conselho Nacional do Desenvolvimento Científico e Tecnológico (CNPq: Proc. 305880/2007-1; 368 304020/2010-9; 573810/2008-7; 575853/2008-5), Fundação de Amparo à Pesquisa do Estado do Amazonas (FAPEAM: Proc. 708565), Instituto Nacional de Pesquisas da Amazônia (INPA: PRJ15.125) and the National Postdoctoral Program (PNPD/CAPES: Proc. No. 028176/2009-41). This article is a contribution of the Brazilian Research Network on Global Climate Change, FINEP/ Rede CLIMA Grant No. 01.13.0353-00.

\section{References}

Adeney, J. M.; Christensen Jr., N. L.; Pimm, S. L. Reserves protect against deforestation fires in the Amazon. PLoS One, 4(4), art. e5014, 2009. doi: 10.1371/journal.pone.0005014 
Aragão, L. E. O. C.; Shimabukuro, Y. E. The incidence of fire in Amazonian forests with implications for REDD. Science, 328, 1275-1278, 2010. doi: 10.1126/science.1186925

Araújo, A. C.; Nobre, A. D.; Kruijt, B.; Culf, A. D.; Stefani, P.; Elbers, J.; Dallarosa, R.; Stefani, P.; von Randow, C.; Manzi, A. O.; Culf, A. D.; Gash, J. H. C.; Valentini, R.; Kabat, P. Dual tower longterm study of carbon dioxide fluxes for a central Amazonian rain forest: The Manaus LBA site. Journal of Geophysical Research, 107(D20), art. 8090. 2002. doi:10.1029/2001JD000676

Arruda, R. "Populações Tradicionais" e a proteção dos recursos naturais em unidades de conservação. Ambiente \& Sociedade, 2(5), 79-92, 1999. Available at: http://www. scielo.br/pdf/asoc/n5/n5a07

Bakkegaard, R. K.; Wunder, S. Bolsa Floresta, Brazil. In: Sills, E. O.; Atmadja, S. S.; de Sassi, C.; Duchelle, A. E.; Kweka, D. L.; Aju, I.; Resosudarmo, P.; Sunderlin, W. D. (Eds.). REDD+ on the ground: A case book of subnational initiatives across the globe. Bogor, Indonesia: Center for International Forestry Research (CIFOR). pp. 51-67. 2014. https://www.cifor.org/redd-case-book/

Barlow, J.; Silveira, J. M.; Mestre, L. A. M.; Andrade, R. B.; D’Andrea, G. C.; Louzada, J.; Vaz-de-Mello, F. Z.; Numata, I.; Lacau, S.; Cochrane, M. A. Wildfires in bamboo-dominated Amazonian forest: Impacts on above-ground biomass and biodiversity. PLoS One, 7(3), art. e33373, 2012. doi: 10.1371/journal.pone.0033373

Barlow, J.; Peres, C. A. Consequences of cryptic and recurring fire disturbances for ecosystem structure and biodiversity in Amazonian forests. In: Laurance, W. F.; Peres, C. A. (Eds.). Emerging Threats to Tropical Forests. Chicago, IL, U.S.A.: University of Chicago Press, pp. 225-240, 2006.

Barlow, J.; Peres, C. A.; Lagan, B. O.; Haugaasen, T. Large tree mortality and the decline of forest biomass following Amazonian wildfires. Ecology Letters, 6(1), 6-8, 2003. doi: 10.1046/j.1461-0248.2003.00394.x

Berenguer, E.; Ferreira, J.; Gardner, T. A.; Aragão, L. E. O. C.; de Camargo, P. B.; Cerri, C. E.; Durigan, M.; de Oliveira Jr., R. C.; Vieira, I. C. G.; Barlow, J. A large-scale field assessment of carbon stocks in human-modified tropical forests. Global Change Biology, 20(12), 3713-3726, 2014. doi: $10.1111 /$ gcb. 12627

Biofílica Investimentos Ambientais AS. Projeto REDD+ Resex Rio Preto-Jacundá. 2016. http://www.biofilica.com. br/web/downloads/jacunda/Plano_Gestao_Jacunda_pt_final.pdf

Brazil, IBAMA. Projeto de Monitoramento do Desmatamento dos Biomas Brasileiros por Satélite - PMDBBS: Cerrado, Monitoramento do Bioma Cerrado 2009-2010. Brasília, DF, Brazil: Instituto Brasileiro do Meio Ambiente e dos Recursos Naturais Renováveis (IBAMA), 2015. Available at: http://siscom.ibama.gov.br/monitora_biomas/ PMDBBS\%20-\%20CERRADO.html

Brazil, IBGE. Manual Técnico da Vegetação Brasileira, $2^{a}$ ed. (Manuais Técnicos em Geociências no. 1). Rio de Janeiro, RJ, Brazil: Fundação Instituto Brasileiro de Geografia e Estatística (IBGE), 271 pp, 2012. Available at: https:// biblioteca.ibge.gov.br/visualizacao/livros/liv63011.pdf

Brazil, INCRA. Download de dados geográficos. Brasília, DF, Brazil: Instituto Nacional de Colonização e Reforma Agrária (INCRA), 2015. Available at: http://acervofundiario.incra.gov.br/i3geo/datadownload.htm

Brazil, INPE. Projeto de Monitoramento do Desmatamento na Amazônia Legal (PRODES). São José dos Campos, SP, Brazil: Instituto Nacional de Pesquisas Espaciais (INPE), 2016. Available at: http://www.dpi.inpe.br/prodesdigital/ prodes.php

Brazil, MMA. Sistema Nacional de Unidades de Conservação (SNUC), Lei no. 9985 de 18 de julho de 2000. Brasília, DF, Brazil: Serviço Brasileiro Florestal (SBF), Ministério do Meio Ambiente (MMA), 2000. Available at: http:// www.mma.gov.br/images/arquivos/areas_protegidas/snuc/ Livro\%20SNUC\%20PNAP.pdf.

Brazil, MMA. Download de dados geográficos. Brasília, DF, Brazil: Ministério do Meio Ambiente (MMA), 2015. Available at: http://mapas.mma.gov.br/i3geo/datadownload.htm

Brazil, Projeto RadamBrasil. Levantamento de Recursos Naturais. Rio de Janeiro, RJ, Brazil: Ministério de Minas e Energia, Departamento Nacional de Produção Mineral, 36 vols., 1973-1983. Available at: https://biblioteca.ibge.gov. br/index.php/bibliote 
Brienen; R. J. W.; Phillips; O. L.; Feldpausch, T. R.; Gloor, E.; Baker, T. R.; Lloyd, J.; Lopez-Gonzalez, G.; Monteagudo-Mendoza, A.; Malhi, Y.; Lewis, S. L.; Martinez, R. V.; Alexiades, M.; Dávila, E. Á.; Alvarez-Loayza, P.; Andrade, A.; Aragão, L. E. O. C.; Araujo-Murakami, A.; Arets, E. J. M. M.; Arroyo, L.; Aymard C., G. A.; Bánki, O. S.; Baraloto, C.; Barroso, J.; Bonal, D.; Boot, R. G. A.; Camargo, J. L. C.; Castilho, C. V.; Chama, V.; Chao, K.-J.; Chave, J.; Comiskey, J. A.; Valverde, F. C.; da Costa, L.; de Oliveira, E. A.; Di Fiore, A.; Erwin, T. L.; Fauset, S.; Forsthofer, M.; Galbraith, D. R.; Grahame, E. S.; Groot, N.; Hérault, B.; Higuchi, N.; Coronado, E. N. H.; Keeling, H.; Killeen, T. J.; Marimon-Junior, W. F.; Mendoza, C.; Neill, D. A.; Nogueira, E. M.; Núñez, P.; Camacho, N. C. P.; Parada, A.; Pardo-Molina, G.; Peacock, J.; Peña-Claros, M.; Pickavance, G. C.; Pitman, N. C. A.; Poorter, L.; Prieto, A.; Quesada, C. A.; Ramírez, F.; Ramírez-Angulo, H.; Restrepo, Z.; Roopsind, A.; Rudas, A.; Salomão, R. P.; Schwarz, M.; Silva, N.; Silva-Espejo, J. E.; Silveira, M.; Stropp, J.; Talbot, J.; ter Steege, H.; Teran-Aguilar, J.; Terborgh, J.; Thomas-Caesar, R.; Toledo, M.; Torello-Raventos, M.; Umetsu, R. K.; van der Heijden, G. M. F.; van der Hout, P.; Vieira, I. C. G.; Vieira, S. A.; Vilanova, E.; Vos, V. A.; Zagt, J. Long-term decline of the Amazon carbon sink. Nature, 519, 344-348. 2015. doi: 10.1038/nature14283

Brown, I. F.; Schroeder, W.; Setzer, A.; Maldonado, M. de los R.; Pantoja, N.; Duarte, A.; Marengo, J. Monitoring fires in southwestern Amazonia rain forests, EOS, Transactions of the American Geophysical Union, 87(26), 253-259, 2006. doi: 10.1029/2006EO260001

Bustamante, M. M. C.; Silva, J. S. O.; Cantinho, R. Z.; Shimbo, J. Z.; Oliveira, P. V. C.; Santos, M. M. O.; Ometto, J. P. H. B.; Cruz, M. R.; Mello, T. R. B.; Godiva, D.; Nobre, C. A. Engagement of scientific community and transparency in $\mathrm{C}$ accounting: the Brazilian case for anthropogenic greenhouse gas emissions from land use, land-use change and forestry. Environmental Research Letters, 13, art. 055005. 2018. doi: 10.1088/1748-9326/aabb37

Carranca, A. Reserva no Acre sofre com falta de fiscalização. O Estado de São Paulo, 4 October 2014, 2014. Available at: http://politica.estadao.com.br/noticias/eleicoes,reserva-no-acre-sofre-com-falta-de-fiscalizacao, 1570568

Cochrane, M. A.; Alencar, A. A.; Schulze, M. D.; Souza,
Jr., C. M.; Nepstad, D. C.; Lefebvre, P.; Davidson, E. A. Positive feedbacks in the fire dynamic of closed canopy tropical forests. Science, 284, 1832-1835, 1999. doi: 10.1126/ science.284.5421.1832

Cox, P. M.; Harris, P. P.; Huntingford, C.; Betts, R. A.; Collins, M.; Jones, C. D.; Jupp, T. E.; Marengo, J. A.; Nobre, C. A. Increasing risk of Amazonian drought due to decreasing aerosol pollution. Nature, 453, 212-215, 2008. doi: 10.1038/nature06960

da Silva, S. S.; Fearnside, P. M.; Graça, P. M. L. A.; Brown, I. F.; Alencar, A.; de Melo, A. W. F. Dynamics of forest fires in the southwestern Amazon. Forest Ecology and Management, 424, 312-322. 2018. doi: 10.1016/j. foreco.2018.04.041

Drummond, J. A. A extração sustentável de produtos florestais na Amazônia brasileira: vantagens, obstáculos e perspectivas. Estudos Sociedade e Agricultura, 6, 115-137, 1996.

ESRI. ArcGIS 10.0. GIS software, Desktop. Redlands, CA, U.S.A.: Environmental Systems Research Institute (ESRI), 2017. http://www.esri.com

Fearnside, P. M. Extractive reserves in Brazilian Amazonia: An opportunity to maintain tropical rain forest under sustainable use. BioScience, 39(6), 387393, 1989. doi: $10.2307 / 1311068$

Fearnside, P. M. Environmental services as a strategy for sustainable development in rural Amazonia. Ecological Economics, 20(1), 53-70, 1997. doi: 10.1016/S09218009(96)00066-3

Fearnside, P. M. Uncertainty in land-use change and forestry sector mitigation options for global warming: Plantation silviculture versus avoided deforestation. Biomass and Bioenergy, 18(6), 457-468. 2000. doi: 10.1016/S09619534(00)00003-9

Fearnside, P. M. Saving tropical forests as a global warming countermeasure: An issue that divides the environmental movement. Ecological Economics,39(2), 167-184, 2001. doi: 10.1016/S0921-8009(01)00225-7

Fearnside, P. M. Time preference in global warming calculations: A proposal for a unified index. Ecological Economics, 41(1), 21-31. 2002. doi: 10.1016/S0921-8009(02)00004-6 
Fearnside, P. M. Conservation policy in Brazilian Amazonia: Understanding the dilemmas. World Development, 31(5), 757-779, 2003. doi: 10.1016/S0305-750X(03)00011-1

Fearnside, P. M. Amazon forest maintenance as a source of environmental services. Anais da Academia Brasileira de Ciências, 80(1), 101-114, 2008. doi: 10.1590/S000137652008000100006

Fearnside, P. M. Carbon benefits from Amazonian forest reserves: Leakage accounting and the value of time. Mitigation and Adaptation Strategies for Global Change, 14(6), 557-567. 2009. doi: 10.1007/s11027-009-9174-9

Fearnside, P. M. Brazil's Amazon forest in mitigating global warming: Unresolved controversies. Climate Policy, 12(1), 70-81, 2012a. doi: 10.1080/14693062.2011.581571

Fearnside, P. M. The theoretical battlefield: Accounting for the climate benefits of maintaining Brazil's Amazon forest. Carbon Management, 3(2), 145-148, 2012b. doi: 10.4155/ CMT.12.9

Fearnside, P. M. What is at stake for Brazilian Amazonia in the climate negotiations. Climatic Change, 118(3), 509-519, 2013. doi: 10.1007/s10584-012-0660-9

Fearnside, P. M. Pesquisa sobre conservação na Amazônia brasileira e a sua contribuição para a manutenção da biodiversidade e uso sustentável das florestas tropicais. In: Vieira, I. C. G.; Jardim, M. A. G.; Rocha, E. J. P. da (Eds.). Amazônia em Tempo: Estudos Climáticos e Socioambientais. Belém, PA, Brazil: Universidade Federal do Pará, Museu Paraense Emílio Goeldi \& Embrapa Amazônia Oriental, pp. 21-49, 2015.

Fearnside, P. M. Brazil's Amazonian forest carbon: The key to Southern Amazonia's significance for global climate. Regional Environmental Change,18(1), 47-61, 2018. doi: 10.1007/s10113-016-1007-2

Fearnside, P. M.; Graça, P. M. L. A. BR-319: Brazil's Manaus-Porto Velho Highway and the potential impact of linking the arc of deforestation to central Amazonia. Environmental Management, 38(5), 705-716, 2006. doi: 10.1007/s00267-005-0295-y

Fearnside, P. M.; Guimarães, W. M. Carbon uptake by secondary forests in Brazilian Amazonia. Forest Ecology and Management, 80(1-3), 35-46, 1996. doi: 10.1016/03781127(95)03648-2

Fearnside, P. M.; Lashof, D. A.; Moura-Costa, P. Accounting for time in mitigating global warming through land-use change and forestry. Mitigation and Adaptation Strategies for Global Change, 5(3), 239-270, 2000. doi: 10.1023/A:1009625122628

Fearnside, P. M.; Yanai, A. M.; Vitel, C. S. M. N. Modeling Baselines for REDD Projects in Amazonia: Is the carbon real? In: Gerold, G.; Jungkunst, H. F; Wantzen, K. M.; Schönenberg, R.; Amorim, R. S. S.; Couto, E. G.; Madari, B.; Hohnwald, S. (Eds.). Interdisciplinary Analysis and Modeling of Carbon-Optimized Land Management Strategies for Southern Amazonia. Göttingen, Germany.Univerditätsdrucke Göttingen, pp. 19-28. 2014. http://webdoc.sub. gwdg.de/univerlag/2014/carbiocial_978-3-86395-138-2.pdf

Feldpausch, T. R.; Lloyd, J.; Lewis, S. L.; Brienen, R. J. W.; Gloor, E.; Mendoza, A. M.; Lopez-Gonzalez, G.; Banin, L.; Abu Salim, K.; Affum-Baffoe, K.; Alexiades, M.; Almeida, S.; Amaral, I.; Andrade, A.; Aragão, L. E. O. C.; Murakami, A. A.; Arets, E. J. M. M.; Arroyo, L., G. A.; Aymard, C.; Baker, T. R.; Bánki, O. S.; Berry, N. J.; Cardozo, N.; Chave, J.; Comiskey, J. A.; Dávila, E. A.; de Oliveira, A.; DiFiore, A.; Djagbletey, G.; Domingues, T. F.; Erwin, T. L.; Fearnside, P. M.; França, M. B.; Freitas, M. A.; Higuchi, N.; E. Honorio C.; Iida, Y.; Jiménez, E.; Kassim, A. R.; Killeen, T. J.; Laurance, W. F.; Lovett, J. C.; Malhi, Y.; Marimon, B. S.; Marimon-Junior, B. H.; Lenza, E.; Marshall, A. R.; Mendoza, C.; Metcalfe, D. J.; Mitchard, E. T. A.; Nelson, B. W.; Nilus, R.; Nogueira, E. M.; Parada, A.; Peh, K. S.-H.; Pena Cruz, A.; Peñuela, M. C.; Pitman, N. C. A.; Prieto, A.; Quesada, C.A.; Ramírez, F.; Ramírez-Angulo, H.; Reitsma, J. M.; Rudas, A.; Saiz, G.; Salomão, R. P.; Schwarz, M.; Silva, N.; Silva-Espejo, J. E.; Silveira, M.; Sonké, B.; Stropp, J.; Taedoumg, H. E.; Tan, S.; ter Steege, H., Terborgh, J., Torello-Raventos, M., van der Heijden, G. M. F., Vásquez, R.; Vilanova, E.; Vos, V.; White, L.; Wilcock, S.; Woell, H.; Phillips, O. L. Tree height integrated into pan-tropical forest biomass estimates. Biogeosciences, 9(8), 3381-3403, 2012. doi: 10.5194/bg-9-1-2012

Gatti, L. V.; Gloor, M.; Miller, J. B.; Doughty, C. E.; Malhi, Y.; Domingues, L. G.; Basso, L. S.; Martinewski, A.; Correia, C. S. C.; Borges, V. F.; Freitas, S.; Braz, R.; An- 
derson, L. O.; Rocha, H.; Grace, J.; Phillips, O. L.; Lloyd, J. Atmospheric $\mathrm{CO} 2$ measurements reveal strong drought sensitivity of Amazonian carbon balance. Nature, 506, 7680, 2014. doi: 10.1038 /nature 12957

GEF - Global Environment Facility. Amazon Region Protected Areas Program. 3rd GEF-7 Replenishment Meeting - Brasília, Brazil, January 23-25, 2018. GEF, World Bank, Washington, DC, USA. 19 pp., 2018, https://www. thegef.org/sites/default/files/publications/Arpa_GEF\%20 2018_22.01.18-v2.pdf

Global Compass. Community monitoring in the Chico Mendes Extractive Reserve in Acre, Brazil. Oxford, UK: Global Canopy Programme (GCP), 2014. Available at: https://forestcompass.org/case-studies/community-monitoring-chico-mendes-extractive-

Gomes, G. A. M. Desafios para Implementação do REDD+ no Brasil: Análise das Ameaças e Oportunidades, Forças e Fraquezas. Doctoral thesis, Salvador, BA, Brazil: Universidade Federal da Bahia, Escola de Administração, 2016. https://repositorio.ufba.br/ri/bitstream/ri/21427/1/ Gomes\%2C\%20Guineverre\%20Alvarez\%20Machado\%20 de\%20Melo.pdf

Grace, J. The Amazon carbon balance: An evaluation of methods and results. In: Nagy, L.; Forsberg, B.; Artaxo, P. (Eds.). Interactions between Biosphere, Atmosphere and Human Land Use in the Amazon Basin. Berlin, Germany: Springer (Ecological Studies 227). pp. 79-100. 2016. doi: 10.1007/978-3-662-49902-3_5

Grupo Carta de Belém. Quem ganha e quem perde com o REDD e Pagamento por Serviços Ambientais? Rio de Janeiro, RJ, Brazil: Fundação Heinrich Böll Brasil, 11 pp. 2011. https://br.boell.org/sites/default/files/downloads/ documento_redd_carta_de_belem_nov_2011.pdf

Heckenberger, M. J.; Kuikuro, A.; Kuikuro, U. T.; Russell, J. C.; Schmidt, M.; Fausto, C.; Franchetto, B. Amazonia 1492: pristine forest or cultural parkland? Science, 301, 1710-1714, 2003. doi: 10.1126/science. 1086112

Heckenberger, M. J.; Russel, C.; Toney, J. R.; Schmidt, M. J. The legacy of cultural landscapes in the Brazilian Amazon: Implications for biodiversity. Philosophical Transactions of the Royal Society, Series B: Biological Sciences, 362,
197-208, 2007. doi: 10.1098/rstb.2006.1979

Homma, A. K. O. História da Agricultura na Amazônia: da Era Pré-Colombiana ao Terceiro Milênio. Brasília, DF, Brazil: Embrapa Informação Tecnológico, Empresa Brasileira de Pesquisa Agropecuária (Embrapa), 274 pp, 2003. Available at: http://ainfo.cnptia.embrapa.br/digital/ bitstream/item/117200/1/HISTORIA-AGRICULTURA-AMAZONIA-Baixa.pdf

Houghton R. A.; Lawrence, K. T.; Hackler, J. L.; Brown, S. The spatial distribution of forest biomass in the Brazilian Amazon: a comparison of estimates. Global Change Biology, 7, 731-746, 2001. doi: 10.1111/j.1365-2486.2001.00426.x

Houghton, R. A.; Skole, D. L.; Nobre, C. A.; Hackler, J. L.; Lawrence, K. T.; Chomentowski, W. H. Annual fluxes of carbon from deforestation and regrowth in the Brazilian Amazon. Nature, 403, 301-304, 2000. doi: 10.1038/35002062

Hutyra, L. R.; Munger, J. W.; Nobre, C. A.; Saleska, S. R.; Vieira, S. A.; Wofsy, S. C. Climatic variability and vegetation vulnerability in Amazônia, Geophysical Research Letters, 32, art. L24712, 2005. doi:10.1029/2005GL024981.

IEF - Instituto Estadual de Florestas do Amapá. Projeto Carbono Cajari. 2018. https://ief.portal.ap.gov.br/dados. php?d $=735 \& \mathrm{a}=4$

Jaramillo-Giraldo, C.; Soares Filho, B.; Ribeiro, S. M.; Gonçalves, R. C. Is it possible to make rubber extraction ecologically and economically viable in the Amazon? The Southern Acre and Chico Mendes Reserve case study. Ecological Economics, 134, 186-197, 2017. doi: 10.1016/j. ecolecon.2016.12.035

Kalamandeen, M.; Gloor, E.; Mitchard, E.; Quincey, D.; Ziv, G.; Spracklen, D.; Spracklen, B.; Adami, M.; Aragão, L. E. O. C.; Galbraith, D. Pervasive rise of small-scale deforestation in Amazonia. Scientific Reports, 8(1), art. 1600, 2018. doi: 10.1038/s41598-018-19358-2

Latif, M.; Semenov, V. A.; Park, W. Super El Niños in response to global warming in a climate model. Climatic Change, 132(4), 489-500, 2015. doi: 10.1007/s10584-015-1439-6

Lewis, S. L.; Phillips, O. L.; Baker, T. R.; Lloyd, J.; Malhi, Y.; Almeida, S.; Higuchi, N.; Laurance, W. F.; Neill, D. A.; Silva, J. N. M.; Terborgh, J.; Lezama, A. T.; Martinez, R. 
V.; Brown, S.; Chave, J.; Kuebler, C.; Vargas, P. N.; Vinceti, B. Concerted changes in tropical forest structure and dynamics: Evidence from 50 South American long-term plots. Philosophical Transactions of the Royal Society of London, Series B: Biological Sciences, 359, 421-436, 2004. doi: $10.1098 /$ rstb.2003.1431

Lewis, S. L.; Brando, P. M.; Phillips, O. L.; van der Heijden, G. M. F.; Nepstad, D. C. The 2010 Amazon drought. Science, 331, 554, 2011. doi: 10.1126/science. 1200807

Lisboa, P. L. Observações sobre a vegetação da campina de areia branca na Amazônia, incluindo revisão bibliográfica. Acta Amazonica, 5(3), 211-223, 1975. http://www.scielo. br/pdf/aa/v5n3/1809-4392-aa-5-3-0211.pdf

Malhi, Y.; Baker, T. R.; Phillips, O. L.; Almeida, S.; Alvarez, E.; Arroyo, L.; Chave, J.; Czimczik, C. I.; Di Fiore, A.; Higuchi, N.; Killeen, T. J.; Laurance, S. G.; Laurance, W. F.; Lewis, S. L.; Montoya, L. M. M.; Agudo, A. M.; Neill, D. A.; Vargas, P. N.; Patiño, S.; Pitman, N. C. A.; Quesada, C. A.; Salomäo, R.; Silva, J. N. M.; Lezama, A. T.; Martínez, R. V.; Terborgh, J.; Vinceti, B.; Lloyd, J. The above-ground coarse wood productivity of 104 Neotropical forest plots. Global Change Biology, 10, 563-591, 2004. doi: 10.1111/j. 1529-8817.2003.00778.x

Malhi, Y.; Doughty, C. E.; Goldsmith, G. R.; Metcalfe, D. B.; Girardin, C. A. J.; Marthews, T. R.; del Aguila-Pasquel, J.; Aragão, L. E. O. C.; Araujo-Murakam, A.; Brando, P.; da Costa, A. C. L.; Silva-Espejo, J. E.; Ameézquita, F. F.; Galbraith, D. R.; Quesada, C. A.; Rocha, W.; Salinas-Revilla, N.; Silvério, D.; Meir, P.; Phillips, O. L. The linkages between photosynthesis, productivity, growth and biomass in lowland Amazonian forests. Global Change Biology, 21, 2283-2295, 2015. doi: 10.1111/gcb.12859

Malhi, Y.; Wood, D.; Baker, T.R.;Wright, J.; Phillips, O. L.; Cochrane, T.; Meir, P.; Chave, J.; Almeida, S.; Arroyo, L.; Higuchi, N.; Killeen, T. J.; Laurance, S. G.; Laurance, W. F.; Lewis, S. L.; Monteagudo, A.; Neill, D. A.; Vargas, P. N.; Pitman. N. C. A.; Quesada, C. A.; Salomão, R.; Silva, J. N. M.; Lezama, A. Torres; Terborgh, J.; Martínez, R. V.; Vinceti, $\mathrm{B}$. The regional variation of aboveground live biomass in old-growth Amazonian forests. Global Change Biology, 12, 1-32, 2006. doi: 10.1111/j.1365-2486.2006.01120.x

Marengo, J. A.; Nobre, C. A.; Tomasella, J.; Oyama, M.
D.; Sampaio de Oliveira, G., Oliveira, R. de; Camargo, H.; Alves, L. M.; Brown, I. F. The drought of Amazonia in 2005. Journal of Climate, 21, 495-516, 2008. doi: 10.1175/2007JCLI1600.1

Marengo, J. A.; Tomasella, J.; Alves, L. M.; Soares, W. R.; Rodriguez, D. A. The drought of 2010 in the context of historical droughts in the Amazon region. Geophysical Research Letters, 38, art. L12703, 2011. doi: 10.1029/2011G1047436

Mazzei, L.; Sist, P.; Ruschel, A.; Putz, F. E.; Marco, P.; Pena. W.; Ferreira, J. E. R. Above-ground biomass dynamics after reduced-impact logging in the Eastern Amazon. Forest Ecology and Management, 259(3), 367-373, 2010. doi: 10.1016/j.foreco.2009.10.031

Moutinho, P.; Stella, O.; Lima, A; Christovam, M.; Alencar, A.; Castro, I.; Nepstad, D. REDD no Brasil: Um Enfoque Amazônico, $3^{\text {a }}$ edição. Brasília, DF, Brazil: Centro de Gestão e Estudos Estratégicos, 156 pp. 2012. http://ipam.org. br/wp-content/uploads/2015/12/redd_no_brasil_um_enfoque_amazônico.pdf

Nascimento, H. E. M.; Laurance, W. F. Total aboveground biomass in central Amazonian rainforests: a landscape-scale study. Forest Ecology and Management, 168(1-3), 311-321, 2002. doi: 10.1016/S0378-1127(01)00749-6

Nepstad, D. C.; Carvalho, G.; Barros, A. C.; Alencar, A.; Capobianco, J. P.; Bishop, J.; Moutinho, P.; Lefebvre, P.; Silva Jr., U. L.; Prins, E. Road paving, fire regime feedbacks, and the future of Amazon forests. Forest Ecology and Management, 154(3), 395-407, 2001. doi: 10.1016/S03781127(01)00511-4

Nepstad, D. C.; Verissimo, A.; Alencar, A.; Nobre, C.; Lima, E.; Lefebvre, P.; Schlesinger, P.; Potter, C.; Moutinho, P.; Mendoza, E., Cochrane, M.; Brooks, V. Large-scale impoverishment of Amazonian forests by logging and fire. Nature, 398, 505-508, 1999. doi: 10.1038/19066

Neves, R. F.; Leal, M. J. L. R.; Vaz, F. Programa de Incentivos a Serviços Ambientais do Carbono do Estado do Acre (Programa ISA Carbono do Acre). Rio Branco, AC, Brazil: IMC. 2013. https://mer.markit.com/br-reg/services/processDocument/downloadDocumentById/103000000029314

Nogueira, E. M.; Fearnside, P. M.; Nelson, B. W.; Barbosa, R. I.; Keizer, E. W. H. Estimates of forest biomass in the 
Brazilian Amazon: New allometric equations and adjustments to biomass from wood-volume inventories. Forest Ecology and Management, 256(11), 1853-1857, 2008a. doi: 10.1016/j.foreco.2008.07.022

Nogueira, E. M.; Fearnside, P. M.; Nelson, B. W.; França, M. B. Wood density in forests of Brazil's 'arc of deforestation': Implications for biomass and flux of carbon from land-use change in Amazonia. Forest Ecology and Management, 248(3), 119-135, 2007. doi: 10.1016/j.foreco.2007.04.047

Nogueira, E. M.; Nelson, B. W.; Fearnside, P. M.; França, M. B.; de Oliveira, Á. C. A. Tree height in Brazil's "arc of deforestation": Shorter trees in south and southwest Amazonia imply lower biomass. Forest Ecology and Management, 255, 2963-2972, 2008b. doi: 10.1016/j.foreco.2008.02.002

Nogueira, E. M.; Yanai, A. M.; Fonseca, F. O. R.; Fearnside, P. M. Carbon stock loss from deforestation through 2013 in Brazilian Amazonia. Global Change Biology, 21(3), 1271-1292, 2015. doi: 10.1111/gcb.12798

Nogueira, E. M.; Yanai, A. M.; Vasconcelos, S. S.; Graça, P. M. L. A.; Fearnside, P. M. Carbon stocks and losses to deforestation in protected areas in Brazilian Amazonia. Regional Environmental Change, 18(1), 261-270, 2018 a. doi: 10.1007/s10113-017-1198-1

Nogueira, E. M.; Yanai, A. M.; Vasconcelos, S. S.; Graça, P. M. L. A.; Fearnside, P. M. Brazil's Amazonian protected areas as a bulwark against regional climate change. Regional Environmental Change, 18(2), 573-579. 2018b. doi: 10.1007/s10113-017-1209-2

Palmer, C.; Taschini, L.; Laing, T. Getting more "carbon bang' for your 'buck' in Acre State, Brazil. Ecological Economics, 142, 214-227. 2017. doi: 10.1016/j.ecolecon.2017.06.024

Ortiz, F. Fundo Amazônia é o único recurso no Brasil para custeio de combate ao desmatamento, diz ISA. OEco, 11 July 2018, 2018. http://www.oeco.org.br/reportagens/ fundo-amazonia-e-o-unico-recurso-no-brasil-para-custeio-de-combate-ao-desmatamento-diz-isa/

Phillips, O. L.; Aragão, L. E. O. C.; Lewis, S. L.; Fisher, J. B.; Lloyd, J.; Lopez-Gonzalez, G.; Malhi, Y.; Monteagudo, A.; Peacock, J.; Quesada, C. A.; van der Heijden, G.; Almeida, S.; Amaral, I.; Arroyo, L.; Aymard, G.; Baker, T.
R.; Banki, O.; Blanc, L.; Bonal, D.; Brando, P.; Chave, J.; de Oliveira, A. C. A.; Cardozo, N. D.; Czimczik, C. I.; Feldpausch, T. R.; Freitas, M. A.; Gloor, E.; Higuchi, N.; Jimenez, E.; Lloyd, G.; Meir, P.; Mendoza, C.; Morel, A.; Neill, D. A.; Nepstad, D.; Patino, S.; Cristina, P. M.; Prieto, A.; Ramirez, F.; Schwarz, M.; Silva, J.; Silveira, M.; Thomas, A. S.; ter Steege, H.; Stropp, J.; Vasquez, R.; Zelazowski, P.; Alvarez, D. E.; Andelman, S.; Andrade, A.; Chao, K.-J.; Erwin, T.; Di Fiore, A.; Honorio, C. E.; Keeling, H.; Killeen, T. J.; Laurance, W. F.; Cruz, A. P.; Pitman, N. C. A.; Nunez, V. P.; Ramirez-Angulo, H.; Rudas, A.; Salamão, R.; Silva, N.; Terborgh, J.; Torres-Lezama, A. Drought sensitivity of the Amazon rainforest. Science, 323, 1344-1347, 2009 b. doi: 10.1126/science.1164033 PMID:19265020

Phillips, O.L.; Baker, T. R.; Arroyo, L.; Higuchi, N.; Killeen, T.; Laurance, W.F.; Lewis, S. L.; Lloyd, J.; Malhi, Y.; Monteagudo, A.; Neill, D. A.; Vargas, P.N.; Silva, J. N. M.; Terborgh, J.; Martinez, R.V.; Alexiades, M.; Almeida, S.; Brown, S.; Chave, J.; Cormiskey, J.A.; Czimczik, C. I.; Fiore, A.D.; Erwin, T.; Kuebler, C.; Laurance, S. G.; Nascimento, H. E M ; Oliveira, J.; Palacios, W.; Patino, S.; Pitman, N. C. A.; Quesada, C. A.; Saldias, M.; Lezama, A. T.; Vinceti, B. Patterns and process in Amazon tree turnover, 1976-2001. Philosophical Transactions of the Royal Society of London, Series B: Biological Sciences, 359, 437-462, 2004. doi: $10.1098 /$ rstb.2003.1438

Phillips, O. L.; Higuchi, N.; Vieira, S.; Baker, T. R.; Chao, K.-J.; Lewis, S.Changes in Amazonian forest biomass, dynamics, and composition, 1980-2002. In: Keller, M.; Bustamante, M.; Gash, J.; da Silva Dias, P. (Eds.). Amazonia and Global Change. Geophysical Monograph Series, Volume 186, Washington, DC, U.S.A.: American Geophysical Union (AGU), pp. 373-387, 2009a. doi: 10.1029/2008GM000739

Quesada, C. A.; Lloyd, J.; Anderson, L. O.; Fyllas, N. M.; Schwarz, M.; Czimczik, C. I. Soils of Amazonia with particular reference to the RAINFOR sites. Biogeosciences, 8 , 1415-1440, 2011. doi: 10.5194/bg-8-1415-2011

Quesada, C. A.; Phillips, O. L.; Schwarz, M.; Czimczik, C. I.; Baker, T. R.; Patiño, S.; Fyllas, N. M.; Hodnett, M. G.; Herrera, R.; Almeida, S.; Alvarez, D. E.; Arneth, A.; Arroyo, L.; Chao, K.-J.; Dezzeo, N.; Erwin, T.; Di Fiore, A.; Higuchi, N.; Coronado, H. E.; Jiménez, E. M.; Killeen, T.; Torres-Lezama, A.; Lloyd, G.; López-Gonzáles, G.; Luizão, 
F. J.; Malhi, Y.; Monteagudo, A.; Neill, D. A.; Vargas, N. P.; Paiva, R.; Peacock, J.; Peñuela, M. C.; Cruz, P. A.; Pitman, N; Priante Filho, N.; Prieto, A.; Ramírez, H.; Rudas, A.; Salomão, R.; Santos, A. J. B.; Schmerler, J.; Silva, N.; Silveira, M.; Vásquez, R.; Vieira, I.; Terborgh, J.; Lloyd, J. Basin-wide variations in Amazon forest structure and function are mediated by both soils and climate. Biogeosciences, 9(6), 2203-2246, 2012. doi: 10.5194/bg-9-2203-2012

Rodrigues, S. Noruega corta $50 \%$ dos repasses para o Fundo Amazônia. OEco, 2 June 2017, 2017. http://www.oeco. org.br/noticias/noruega-corta-50-dos-repasses-para-o-fundo-amazonia/

Saatchi, S, S.; Houghton, R. A.; dos Santos Alvala, R. C.; Soares. J. V.; Yu, Y. Distribution of aboveground live biomass in the Amazon Basin. Global Change Biology, 13, 816-837, 2007. doi: 10.1111/j.1365-2486.2007.01323.x

Salazar, L. F.; Nobre, C. A.; Oyama, M. D. Climate change consequences on the biome distribution in tropical South America. Geophysical Research Letters, 34, art. L09708, 2007. doi: 10.1029/2007GL029695

Salisbury, D. S.; Schmink, M. Cows versus rubber: changing livelihoods among Amazonian extractivists. Geoforum, 38(6), 1233-1249, 2007. doi: 10.1016/j.geoforum.2007.03.005

Salomon, M. Gado avança em reserva Chico Mendes. Folha de São Paulo, 21 September 2008, 2008. Available at: http:// www1.folha.uol.com.br/fsp/brasil/fc2109200819.htm

Silva, S. S. da; Alencar, A. A.; Mendoza, E.; Brown, I. F. Dinâmica dos incêndios florestais no Estado do Acre nas décadas de 90 e 00. In: Estiphanio, J. C. N.; Galvão, L. S. (Eds.). Simpósio Brasileiro de Sensoriamento Remoto (SBSR), 16. 2013, Foz do Iguaçu, Anais. São José dos Campos, SP, Brazil: Instituto Nacional de Pesquisas Espaciais (INPE), pp. 8799-8806, 2013. Available at: http://urlib. net/3ERPFQRTRW34M/3E7GLQ6

Silva, S. S. da; Graça, P. M. L. A.; Numata, I.; Ferreira, E. J. L.; Fearnside, P. M.; Santos, E. A. dos; de Lima, R. C.; Brown, I. F. Incêndios florestais como fator de mudança na dominância do bambu em florestas abertas no leste do Acre. In: XVIII Simpósio Brasileiro de Sensoriamento Remoto, Santos-SP, 28 a 31 de maio de 2017. São José dos Campos,
SP, Brazil: Sociedade Brasileira de Sensoriamento Remoto (SBSR), Instituto Nacional de Pesquisas Espaciais (INPE), pp. 5605-5611, 2017. Available at: https://proceedings. galoa.com.br/sbsr/trabalhos/incendios-florestais-como-fator-de-mudanca-na-dominancia-do-bambu-em-florestas-abertas-no-leste-do

Sist, P.; Ferreira, F. N. Sustainability of reduced-impact logging in the eastern Amazon. Forest Ecology and Management, 243, 199-209, 2007. doi: 10.1016/j.foreco.2007.02.014

Sist, P.; Mazzei, L.; Lilian, L.; Rutishauser, E. Large trees as key elements of carbon storage and dynamics after selective logging in the Eastern Amazon. Forest and Ecology and Management, 318, 103-109, 2014. doi: 10.1016/j. foreco.2014.01.005

Soares-Filho, B. S. Role of Amazon protected areas, especially the conservation units supported by ARPA, in reducing deforestation. Fundo Brasileiro para Biodiversidade (Funbio), Rio de Janeiro, RJ, Brazil. 12 pp. 2016. https:// www.funbio.org.br/wp-content/uploads/2018/02/Role-of-Amazon-Protected-Areas_IN.pdf

Soares-Filho, B. S.; Moutinho, P.; Nepstad, D.; Anderson, A.; Rodrigues, H.; Garcia, R.; Dietzsch, L.; Merry, F.; Bowman, M.; Hissa, L.; Silvestrini, R.; Maretti. C. Role of Brazilian Amazon protected areas in climate change mitigation. Proceedings of the National Academy of Sciences USA 107(24), 10,821-10,826, 2010. doi: 10.1073/ pnas.0913048107

Stickler, C. M.; Nepstad, D. C.; Coe, M. T.; McGrath, D. G.; Rodrigues, H. O.; Walker, W. S.; Soares-Filho, B. S.; Davidson, E. A. The potential ecological costs and cobenefits of REDD: a critical review and case study from the Amazon region. Global Change Biology,15,2803-2824, 2009. doi: 10.1111/j.1365-2486.2009.02109.x

Superti, E.; Aubertin, C. Pagamentos por serviços ambientais na Amazônia: O desvio de um conceito - casos do Amapá e Acre. Desenvolvimento e Meio Ambiente, 35, 209-224. 2015. doi: 10.5380/dma.v35i0.38976

Uhl, C.; Buschbacher, R. A disturbing synergism between cattle-ranch burning practices and selective tree harvesting in the eastern Amazon. Biotropica, 17(4), 265-268, 1985. 
doi: $10.2307 / 2388588$

Vadjunec, J. M.; Gomes, C. V. A.; Ludewigs, T. Lan$\mathrm{d}$-use/land-cover change among rubber tappers in the Chico Mendes Extractive Reserve, Acre, Brazil. Journal of Land Use Science, 4(4), 249-274, 2009. doi: $10.1080 / 17474230903222499$

Vasconcelos, S. S.; Fearnside, P. M.; Graça, P. M. L. A.; Nogueira, E. M.; Oliveira, L. C. de; Figueiredo, E. O. Forest fires in southwestern Brazilian Amazonia: Estimates of area and potential carbon emissions. Forest Ecology and Management, 291, 199-208, 2013. doi: 10.1016/j. foreco.2012.11.044

Veríssimo, A.; Rolla, A.; Vedoveto, M.; Furtada, S. M. Áreas Protegidas na Amazônia Brasileira: Avanços e Desafios. Belém, PA, Brazil: Instituto do Homem e Meio-Ambiente da Amazônia (IMAZON) \& São Paulo, SP, Brazil: Instituto Sócio Ambiental (ISA) 87pp, 2011. Available at: https:// www.socioambiental.org/sites/blog.socioambiental.org/ files/publicacoes/10372_0.pdf

Viana, V.; Tezza, J.; Solidade, V.; Marostica, S.; Salviati, V.; Soares, A. Impactos do Programa Bolsa Floresta: Uma avaliação preliminar. Inclusão Social, 6(1), 201-218, 2012. http://revista.ibict.br/inclusao/article/view/1703

Vitel, C. S. M. N.; Carrero, G. C.; Cenamo, M. C.; Leroy, M.; Graça, P. M. L. A.; Fearnside, P. M. Land-use change modeling in a Brazilian indigenous reserve: Construction a reference scenario for the Suruí REDD project. Human Ecology, 41(6), 807-826, 2013. doi: 10.1007/s10745-0139613-9

Wandelli, E. V.; Fearnside, P. M. Secondary vegetation in central Amazonia: Land-use history effects on aboveground biomass. Forest Ecology and Management, 347, 140-148, 2015. doi: 10.1016/j.foreco.2015.03.020

World Bank. Rainforest Trust Fund Resolution, Background note, Part I: IBRD Resolution 92-2 (24 March 1992) Introduction and Objectives. World Bank, Washington, DC, USA, Available at http://www.worldbank.org.

World Bank. Report No. 13047-BR Pilot Program to Conserve the Brazilian Rain Forest Memorandum and Recommendation. World Bank, Washington, DC, USA, 1994. http://documents.worldbank.org/curated/ en/231801468769129079/text/multi-page.txt

Wunder, S.; Börner, J.; Tito, M. R.; Pereira, L. Pagamentos por Serviços Ambientais: Perspectivas para a Amazônia Legal, $2^{\mathrm{a}}$ ed. MMA, Brasília, DF, Brazil: Ministério do Meio Ambiente (MMA) (Série Estudos, 10), 141 pp, 2009. Available at: http://www.mma.gov.br/estruturas/168/_publicacao/168_publicacao17062009123349.pdf

Yanai, A. M.; Fearnside, P. M.; Graça, P. M. L. A.; Nogueira, E. M. Avoided deforestation in Brazilian Amazonia: Simulating the effect of the Juma Sustainable Development Reserve. Forest Ecology and Management, 282, 78-91, 2012. doi: 10.1016/j.foreco.2012.06.029

Zeng, N.; Yoon, J. -H.; Marengo, J. A.; Subramaniam, A.; Nobre, C. A.; Mariotti, A.; Neelin, J. D. Causes and impacts of the 2005 Amazon drought. Environmental Research Letters, 3, art. 014002, 2008. doi: 10.1088/17489326/3/1/014002 